Omega-P, Inc.

Final Report on 2010 SBIR Phase I grant DE-SC-0004491

SHORT-PERIOD RF UNDULATOR FOR A SASE NANOMETER SOURCE

Omega-P, Inc.

291 Whitney Avenue, Suite 401

New Haven, CT 06511

Final Report to US Department of Energy

SBIR Phase I Grant DE-SC000 4491

\title{
SHORT-PERIOD RF UNDULATOR FOR A SASE NANOMETER SOURCE
}

\section{CONTENTS}

$\begin{array}{ll}\text { I. INTRODUCTION } & 2\end{array}$

II. TECHNICAL APPROACH 4

IIa. Technical background $\quad 4$

IIb. Concepts for a mm-wave RF undulator 5

IIc. Electron orbits for a beam traversing a microwave resonator 6

IId. Microwave undulator resonator designs 15

$\begin{array}{ll}\text { III. CONCLUSIONS } & 28\end{array}$

$\begin{array}{ll}\text { References } & 30\end{array}$

\section{SUMMARY}

Analysis is described towards development of a RF undulator with a period $<1 \mathrm{~cm}$, an undulator parameter $K$ of the order of unity, and a gap greater than $2.25 \mathrm{~mm}$. The application for the undulator is for a SASE source to produce $1 \mathrm{~nm}$ wavelength radiation using a low energy electron beam in the range 1-2 GeV. Particle orbit calculations in a conventional standing-wave resonator configuration show that the presence of a co-propagating component of RF field can cause deleterious motion for the undulating electrons that can seriously degrade their radiation spectrum. To obviate this problem, resonator designs were devised in which only the counterpropagating field components interact with the particles. Two resonator configurations with the same undulator parameter $K=0.4$ have been devised and are described in this report.

Material presented in this report is the result of work by Omega-P Senior Research Physicists Dr. S.V. Kuzikov and Dr. G. Sotnikov, Senior Consultant Professor T.C. Marshall, with Dr. J.L. Hirshfield serving as Principal Investigator.

The proposal was written by Professor Marshall and Dr. Hirshfield.

December 19, 2011 


\section{Omega-P, Inc.}

Final Report on 2010 SBIR Phase I grant DE-SC-0004491

\section{SHORT-PERIOD RF UNDULATOR FOR A SASE NANOMETER SOURCE}

\section{INTRODUCTION}

Under Topic 15c in the DoE 2010 SBIR Program Solicitation, Phase I proposals were sought for development of undulators for current and future light sources. Item (6) under this topic in the Solicitation specifically requested proposals to develop an undulator with period $<1$ $\mathrm{cm}$, with $K$-value* of the order of unity, and with a gap greater than $2.25 \mathrm{~mm}$. The application for the undulator cited in the Solicitation is for a SASE source to produce $1 \mathrm{~nm}$ wavelength radiation using a low energy electron beam in the range 1-2 GeV. Omega-P, Inc. submitted a proposal entitled "Short period RF undulator for a SASE source," and was awarded Phase I grant DE-SC0004491, for the period 6/19/10 - 3/18/11. The present document gives results of the Phase I study. The follow-on Phase II grant was not approved. Phase II had been planned to culminate in the construction and testing of an RF undulator driven by the Omega-P/Yale 34.3 $\mathrm{GHz}$ magnicon having an undulation period of $0.44 \mathrm{~cm}$, a $>1 \mathrm{~cm}$ gap, and-depending upon available RF power - a $K$-value of the order of unity. It was not to be a principal goal of the Phase II project to obtain FEL radiation from a relativistic beam driven by the RF undulator. However, the undulator could have been built to accommodate a $6-\mathrm{MeV}$ beam from an available RF gun at the Yale University Beam Physics Lab and attempts would have been made to detect micron-wavelength spontaneous emission from the beam so as to assess the properties of the undulator.

The Phase I studies confirm our preliminary speculation that an RF undulator could satisfy criteria set forth in the 2010 Solicitation. For one of the point designs described in this report, the effective undulator period is $0.45 \mathrm{~cm}$, the undulator parameter* is $K=0.4$, and the effective gap is 10 's of $\mathrm{mm}$. These remarkable undulator parameters - as yet not optimized - are expected to be realized in the laboratory because of the availability of multi-MW RF power from the Omega-P/Yale pulsed magnicon amplifier operating at $34.3 \mathrm{GHz}$ to drive the undulator, and upon sophisticated computational tools for designing a room-temperature copper structure that can support the necessary strong RF fields. Production of $1 \mathrm{~nm}$ radiation using an undulator with these parameters requires the electron beam energy to be only about $0.5 \mathrm{GeV}$, significantly lower than the $1-2 \mathrm{GeV}$ beam cited in the Solicitation; this greatly lowered electron beam energy should translate into considerable cost savings for the light source.

Adoption of room-temperature RF technology for strong-field, short-period undulators may allow parameter ranges for a future light source to be realized that are not readily accessible with conventional magnets. Savings in accelerator size and cost if the required beam energy for

\footnotetext{
*The conventional definition [1] is used here for $K$, namely $K=e B \lambda / 2 \pi m c=93.4 B[\mathrm{~T}] \times \lambda[\mathrm{m}]$, where $B$ is the maximum transverse magnetic field and $\lambda$ depends upon the type of RF mode. In an RF undulator, both transverse electric and magnetic fields may contribute to the wiggling motion needed for an FEL. For traveling waves, $\lambda=\lambda_{0}$ the free-space wavelength if the guided wave is TM; and $\lambda=\lambda_{\mathrm{g}}$ the guide wavelength if it is TE. Using this definition, the normalized transverse velocity $\beta_{\perp}$ of an undulating electron is $K / \gamma$, as for a conventional magnetic undulator.
} 


\section{SHORT-PERIOD RF UNDULATOR FOR A SASE NANOMETER SOURCE}

the accelerator is reduced by half or more could also be considerable. The trade-off for these attractive features includes need for a substantial RF installation to power the undulator, including numbers of RF sources, modulators, and RF distribution systems. How these virtues and costs balance out for a given light source could be judged in practice after a proof-ofprinciple prototype of the strong-field, short-period RF undulator is built and evaluated.

In the course of conducting the Phase I study, a deficiency in the "conventional wisdom" regarding RF undulators was uncovered. This discovery was revealed during studies of electron orbit dynamics in an undulator configured as a standing-wave resonator [2,3]. Resonators have been long proposed to sustain the high amplitude RF magnetic fields that induce the undulatory motion of electrons on a beam traversing the resonator, and examples of several resonators were given in our Phase I proposal. A standing wave can be viewed as comprising two equal amplitude waves traveling in opposing directions. The wave traveling opposite to the direction of the beam (hereafter the counter-propagating wave) is the component that induces the desired quiver motion that underlies the FEL process [4]. But the wave travelling in the same direction as the beam (the co-propagating wave) will also induce motions - of different periodicity and of much larger amplitude. These latter motions will indirectly influence the radiation spectrum in the FEL by causing a strong distortion of electron beam orbits, leading to unacceptable emittance growth in the beam as it traverses the undulator, with a profound effect upon the radiation spectrum. The deleterious influence of the co-propagating wave upon the spectrum was overlooked in the otherwise preeminent work by Shintake [5] that first described observations of spontaneous radiation from a beam traversing a strongly-pumped microwave resonator.

It would appear that this deficiency can be circumvented by using a resonant ring, in which only the counter-propagating wave would be present. In fact, a mm-wave quasi-optical resonant ring with an effective power gain of 35:1 was built and demonstrated by Omega-P and Institute of Applied Physics scientists in 2005 [10]. This structure has further appeal for a highpower RF undulator since fields on the walls have half the values that would prevail for a similar standing wave resonator supporting the same undulator $K$-parameter. This latter argument could be promulgated until now even without knowledge of the more fundamental issue with orbit distortions due to a co-propagating wave. Unfortunately, as will be described in detail in Section III, the price to be paid for these attributes of a resonant ring is demand for a very high level of RF power to achieve a $K$-parameter above about 0.2 ; such power level requirements might derail the resonant ring as a candidate for this application.

Fortunately, a pathway around this difficulty has been found. During the Phase I study, Omega-P senior research physicist S.V. Kuzikov devised a class of standing-wave resonatorsdescribed below-where the co-propagating wave component is almost fully decoupled from the electron beam, and only the counter-propagating wave wiggles the particles. This is achieved through use of mode converters at the ends of the resonator that transform a mode with strong coupling to the beam, to a second mode where the coupling is nearly absent. Calculations 
Omega-P, Inc.

Final Report on 2010 SBIR Phase I grant DE-SC-0004491

\section{SHORT-PERIOD RF UNDULATOR FOR A SASE NANOMETER SOURCE}

described below reveal that this type of dual-mode resonator can provide a given $K$-parameter over a fixed undulator length using only a fraction of the power required for a corresponding resonant ring.

\section{TECHNICAL APPROACH}

\section{IIa. Technical background}

The idea of an RF undulator is decades old, and expositions on its underlying physics can be found in several books, e.g., [4], often with the label "optical wiggler," since intense lasers were among the radiation sources considered as drivers for such undulators. The basic idea is to substitute transverse axially-periodic RF magnetic fields for transverse axially-periodic DC magnetic fields, either with linear or helical polarization. In the RF case, the frequency up-shift for Compton back-scattered photons $\sim 4 \gamma^{2}$ can be twice the up-shift for the DC case $\sim 2 \gamma^{2}$ between the frequency of undulation $\sim c / \lambda$ and the frequency for peak FEL or SASE gain [1]. Here, $\gamma=1+W / m c^{2}$ is the relativistic energy factor for electrons with energy $W$. The factor-

of-two difference in up-shift can translate into a reduction by a factor of $\sqrt{2}$ in the energy of the electron beam in a light source designed to operate down to a given wavelength.

Perhaps the earliest demonstration of an RF undulator was by T. Shintake et al [5]. That device employed a meter-long $\mathrm{TE}_{1,0,19}$-mode cavity that used rectangular ridged waveguide, which when driven with $300 \mathrm{~kW}$ of RF power at $2856 \mathrm{MHz}$ generated an equivalent undulator magnetic field of $0.043 \mathrm{~T}$, an undulator period of $55 \mathrm{~mm}$, and an undulator parameter $K$ of 0.24 ; undulator radiation in the visible was observed using a $143 \mathrm{MeV}$ electron beam. However, we have found that Shintake overlooked the mechanism that can call into question the usefulness of his result, namely the deleterious influence of the co-propagating wave mentioned above. M. Seidel [6] cataloged equations and parameters for a wide range of RF waveguide-based undulators, and discussed a number of practical options for creating RF fields in a waveguide strong enough to achieve practical undulator parameters, based on then-available RF sources. He considered several options for normal and superconducting resonant rings and pointed out limitations for undulator parameters and undulator wavelengths. In [2], Pelligrini described an $\mathrm{X}$-band undulator based on use of square waveguide operating with a rotating TE mode. This device requires an RF power level of $800 \mathrm{MW}$ to provide an undulator parameter $K=0.4$ with an undulator period of $1.45 \mathrm{~cm}$. X-band power at this level has been produced using four 50MW pulsed klystrons and a SLED-II pulse compressor [7]. The idea of an RF undulator was further amplified upon by Tantawi et al [3], who pointed out the merits of designing the device based on use of the $\mathrm{TE}_{12}$ mode in circular (or even elliptic) waveguide, as a means of reducing $\mathrm{RF}$ fields on the waveguide walls which, at the required power levels using the $\mathrm{TE}_{11}$ mode, might not be sustainable due to RF breakdown and surface fatigue. 


\section{SHORT-PERIOD RF UNDULATOR FOR A SASE NANOMETER SOURCE}

The undulator parameter $K=e B \lambda / 2 \pi m c=93.4 B[\mathrm{~T}] \times \lambda[\mathrm{m}]$ is a key dimensionless quantity determining SASE radiation intensity [1]. In RF undulators, $K$ is limited by these factors:

a. surface electric fields, that lead to breakdown;

$b$. surface magnetic fields that lead to quenching in $\mathrm{SC}$ waveguides, and to metal fatigue in room-temperature (warm) waveguides;

c. ohmic power losses in waveguide walls; and ultimately

$d$. the peak power levels available from RF sources.

Items $c$ and $d$ are the most severe of these as one attempts to shorten the undulator period, to say less than $1 \mathrm{~cm}$, since a short period RF undulator requires high power RF at frequencies of 10's of $\mathrm{GHz}$ where Ohmic losses are high and high-power RF sources are rare.

In this report, an RF undulator is described that allows one to achieve a relatively high undulator parameter, i.e. $K \approx 0.4$, at the same time as a small undulator period, namely $0.87 \mathrm{~cm}$, using a presently-available RF source. Gap size would be the cavity diameter, some 10's of mm. The RF source to be used is the Omega-P/Yale $34 \mathrm{GHz}$ magnicon, designed for a power of 20 MW or more at pulse widths up to $1 \mu \mathrm{s}$; at a maximum repetition rate of $10 \mathrm{~Hz}$. A photograph of the magnicon installed and operating in the Yale Beam Physics Lab is shown in Fig. 1.

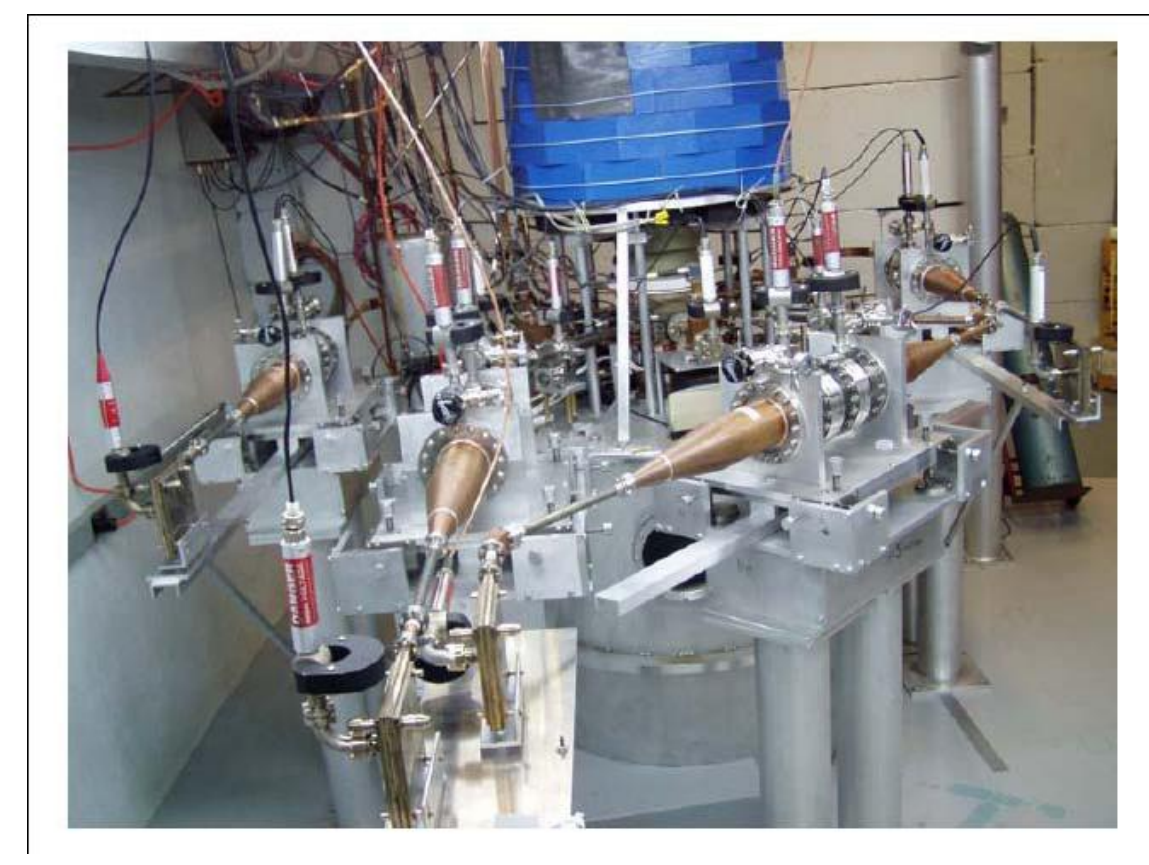

Fig. 1. Photograph of Ka-band magnicon test facility at Yale Beam Physics Lab.

\section{IIb. Concepts for a mm-wave RF undulator}

In this section we describe work done during Phase I, aimed towards use of a microwave resonator to set up the FEL interaction on a bunch of relativistic electrons traversing the resonator. The analysis to be described is general, in the sense that it can inform design of an RF 


\section{SHORT-PERIOD RF UNDULATOR FOR A SASE NANOMETER SOURCE}

undulator to meet a range of requirements, but with examples that can meet the specifications given in the DoE 2010 Solicitation. These in turn are based on parameters of the multi-MW 34$\mathrm{GHz}$ magnicon amplifier and the 6-MeV RF gun and beamline available at the Yale Beam Physics Laboratory; these facilities could have been available for proof-of-principle experiments under a sub-grant from Omega-P to Yale University, had the Phase II grant been approved.

For the proof-of-principle experiments, we proposed to use the high power magnicon that has shorter wavelength $(8.77 \mathrm{~mm})$ than existing powerful X-band klystrons $(27 \mathrm{~mm})$ anticipated for this application by others [2,3], notwithstanding the lower power of the magnicon. The shorter wavelength permits one to reduce the beam energy required to generate nm wavelengths, but the lower power of the magnicon requires that the microwave fields build up amplitude in a resonator so that $K \sim 0.4$ can be obtained. This $K$-value is limited by pulsed heating capabilities of the structure, surface breakdown at the walls, and available power from a short-wavelength microwave source--problems that affect all RF undulator designs.

So as to establish the need for an RF structure that supports a counter-propagating wave to couple with the electron beam, and a co-propagating wave that does not, Section IIb presents results of computing electron orbits in each of these waves. Section IIc presents designs and operating parameters for a dual-mode resonator and a resonant ring resonator, together with a comparison of their parameters for equal undulator performance. Results of the Phase I study are summarized in Section III.

\section{IIc. Electron orbits for a beam traversing a microwave resonator}

It may appear that use of a resonator is a straightforward approach for building an RF undulator. But the standing-wave nature of the RF fields introduce a different issue for FEL operation, as we found while carrying out the Phase I project; but thankfully, there is a solution to these difficulties, as we shall also show in Section IIc. Unlike a strictly traveling-wave RF undulator, where a single intense high frequency wave moves in a waveguide counter to the motion of an electron bunch, there is in a conventional resonator a companion wave which moves parallel to the bunch. We have found that this co-propagating wave causes mischief with the beam, requiring a special design to overcome this fault. It is the counter-propagating wave, as is easily shown [1,4], that causes the desired FEL quiver motion of the electrons in the RF undulator and the relativistic Doppler upshift of the radiation wavelength, as described in Section I of this proposal. The forward wave interacts with the electrons and generates low frequency microwave radiation, which though not harmful, is part of a process that disturbs the beam, causing a spatially-growing lateral motion. It seems that this effect has not been properly understood and appreciated in the past.

In 1987, Tran, Danly, and Wurtele [8] examined the standing wave resonator concept. They studied counter-propagating plane waves, which have constant amplitude across the plane transverse to the particle motion, and which have only transverse fields. Their analysis found 
Omega-P, Inc.

Final Report on 2010 SBIR Phase I grant DE-SC-0004491

\section{SHORT-PERIOD RF UNDULATOR FOR A SASE NANOMETER SOURCE}

that, for $K \sim 1$, the radiation spectrum splits into many separate modes because the motion of the electrons contains many harmonics. The spectrum they computed is shown in Fig. 2, and the behavior of some of the harmonic coefficients $f_{\mathrm{n}, \mathrm{m}}$ as $K$ is increased is shown in Figs. 3. Due to the co-propagating wave, the electron axial motion is not constant and this gives rise to the harmonic spectrum; but as we shall see, there are other important effects too. From Fig. 2, it might appear that if we operated the device using $K<0.5$, we might avoid these complications; but unfortunately that has been found to be not altogether correct.

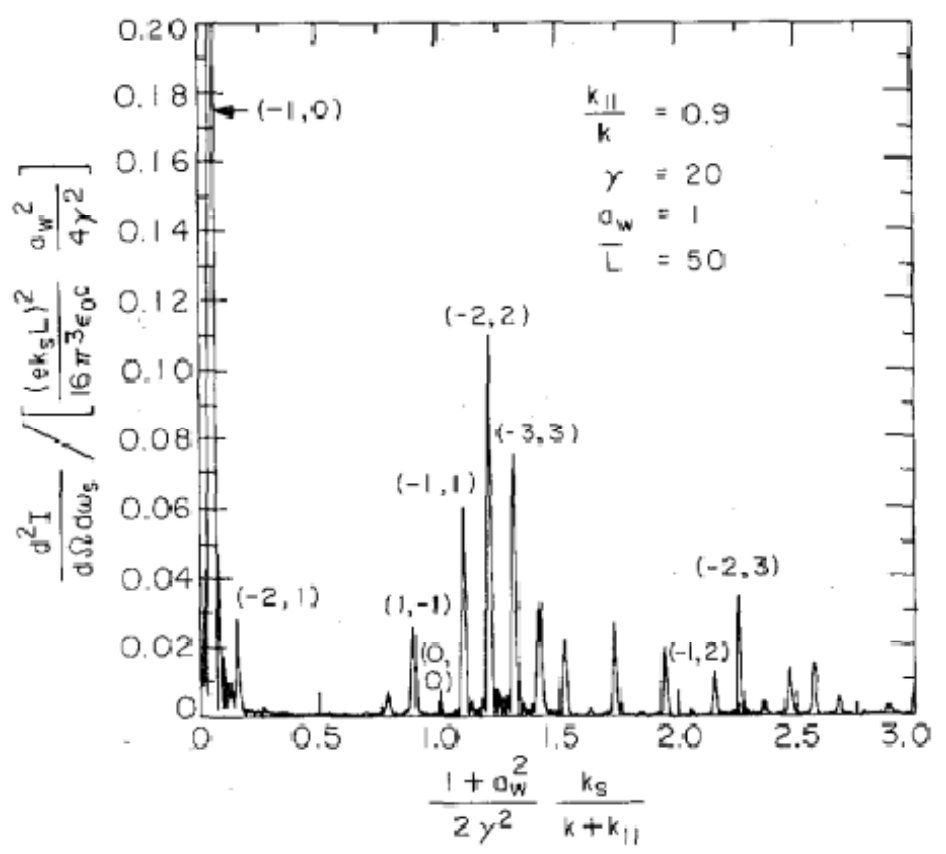

Fig. 2. [From ref. 8] Normalized spontaneous emission spectrum versus frequency for a linearly-polarized RF undulator for $K\left(a_{\mathrm{w}}\right.$ here $)=1$. This example is similar to our case. The

FEL radiation is from the $(0,0)$ mode, the low frequency radiation is the $(-1,0)$ mode.

The FEL peak has almost disappeared. (C) IEEE, 1987.

We begin our study with an example with a resonator in which standing waves are set up at $34.27 \mathrm{GHz}$ in the $\mathrm{TE}_{01}$ (circular-symmetric) mode. This mode has favorable properties regarding RF breakdown. A map of its radial rf magnetic field is shown in Fig. 4, with its maximum value at $0.48 R$, where $R$ is the cavity radius. 
Omega-P, Inc.

Final Report on 2010 SBIR Phase I grant DE-SC-0004491

SHORT-PERIOD RF UNDULATOR FOR A SASE NANOMETER SOURCE

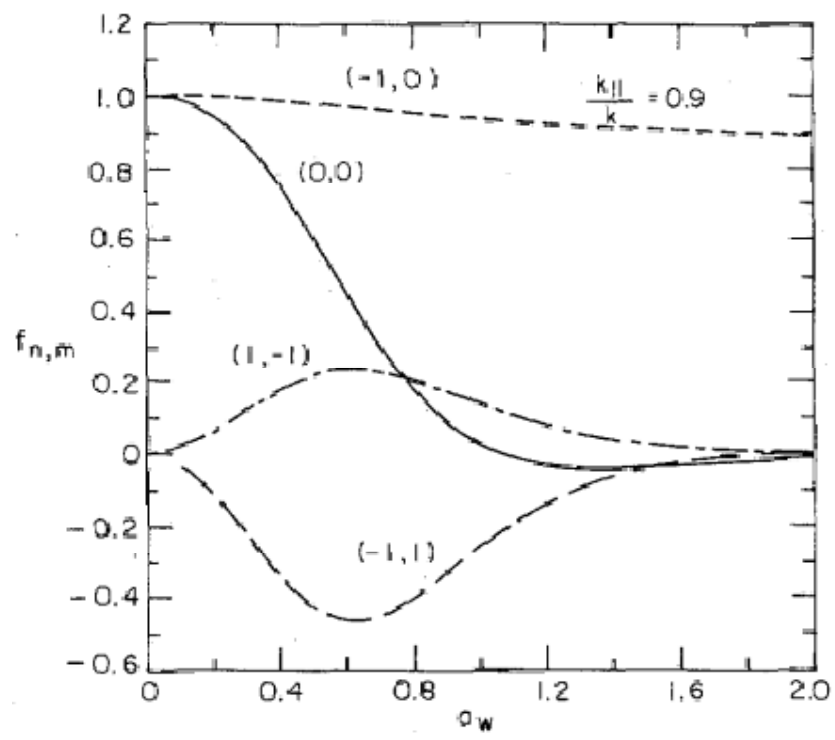

Fig. 3. [From ref. 8] Coefficients $f_{\mathrm{n}, \mathrm{m}}$ of the harmonics of the motion versus undulator parameter $a_{\mathrm{w}}[8]$ for a linearly-polarized rf undulator; $(0,0)$ is the desired mode. (C) IEEE, 1987.
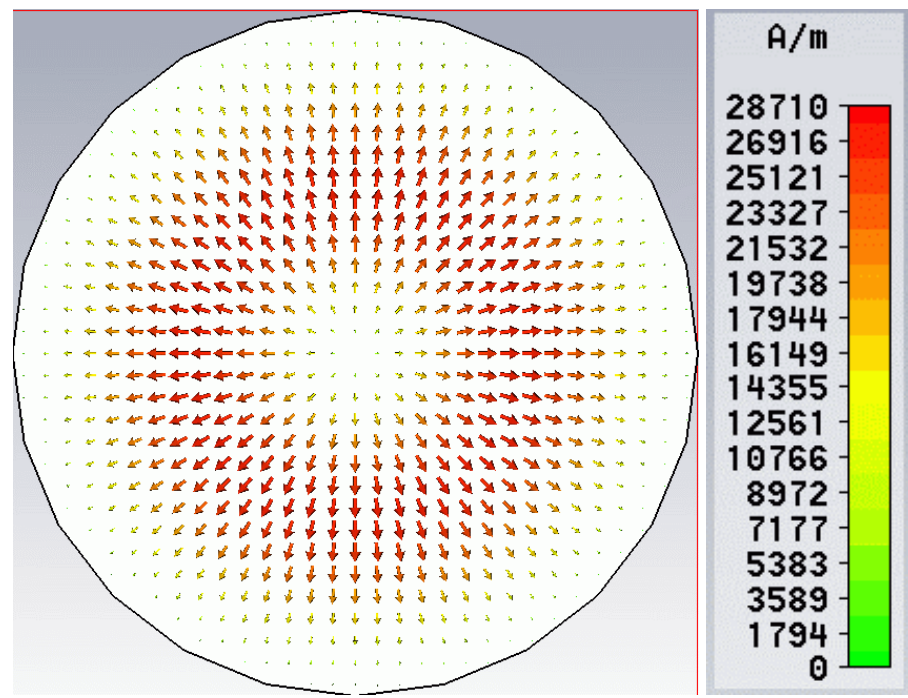

Fig. 4. 2D plot of the transverse magnetic field intensity for the $\mathrm{TE}_{01}$ mode in cross-section in a cylindrical resonator.

The motion of a filamentary beam in fields of this resonator has been studied, using the CST Studio code. A filament is chosen to simplify depiction of the orbit. This code does not have the capability to handle a resonator; instead, we launch counter-propagating waves from each end, and compute orbits during the one-way pass of each wave component. The wave power is artificially chosen to achieve the desired value of $K$. The beam is located off-axis, at $r$ $=6.5 \mathrm{~mm}$ where the transverse fields peak. We take the beam energy to be $6 \mathrm{MeV}$, the 


\section{SHORT-PERIOD RF UNDULATOR FOR A SASE NANOMETER SOURCE}

waveguide radius $14 \mathrm{~mm}$, the length of the system $50 \mathrm{~cm}$, and the (effective) $\mathrm{RF}$ power $=200$ MW. Fig. 5 shows a projection of the beam orbit in the $z, x$ plane. Colors represent different beam energies, red being $6.012708 \mathrm{MeV}$ and dark blue being $5.98377 \mathrm{MeV}$, a range of $29 \mathrm{keV}$. Not only is this energy modulation far in excess of what is expect for the FEL quiver energy of the electrons (a few hundred $\mathrm{eV}$ at most), but there is a spatially-growing oscillation that has an amplitude on the mm scale, also not a FEL-induced motion of the particles. This we identify to be an instability. The transverse view along the beam axis in Fig. 6 shows this motion in the $x, y$ plane.
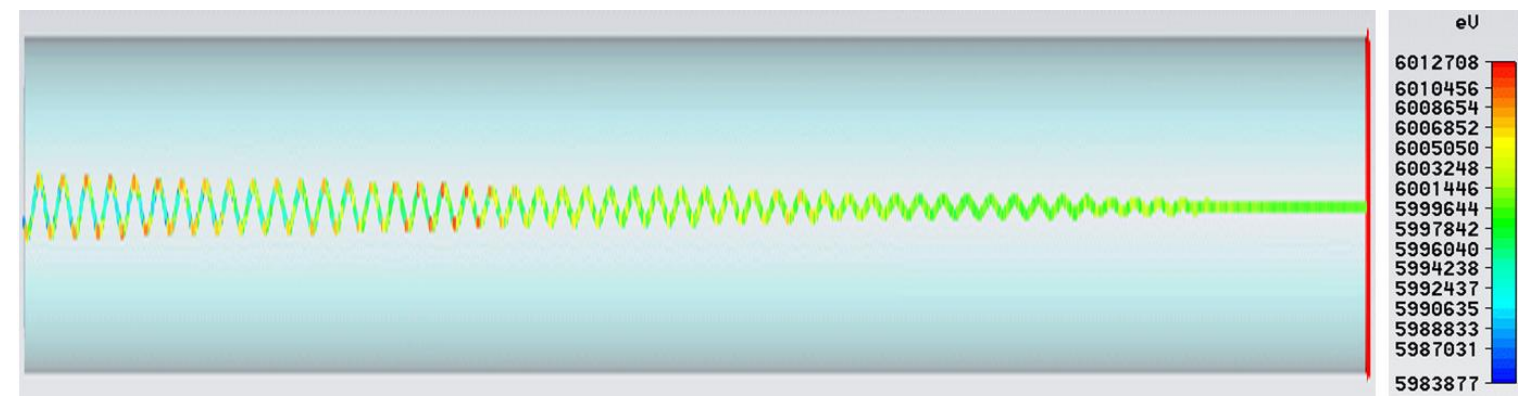

Fig. 5. Electrons travel from right to left in a co-propagating wave. The radial magnetic field of the $\mathrm{TE}_{01}$ wave at the beam location is $0.11 \mathrm{~T}$, and $K=0.1$.

Transverse dimensions are enhanced to better show beam motions.

One notes the growing amplitude of transverse motion.

Fig. 6. Beam oscillation seen in the $x, y$ plane ( $x$ is horizontal). The radial field is here $0.35 \mathrm{~T}$ corresponding to $K=0.31$. The beam is located off-axis at $y=6.5 \mathrm{~mm}$.

In order to understand this phenomenon, we first endeavor to see the electron quiver motion, driven by the RF undulator, that would itself give rise to the spontaneous FEL radiation. The parameters of the study are provided in Table I. The FEL quiver motion has a normalized 
Omega-P, Inc.

Final Report on 2010 SBIR Phase I grant DE-SC-0004491

\section{SHORT-PERIOD RF UNDULATOR FOR A SASE NANOMETER SOURCE}

amplitude $\beta_{\mathrm{x}}=K / \gamma$, so $P_{\mathrm{x}} / \gamma m c=K=0.05$ in the isolated counter-propagating $200 \mathrm{MW}$ wave we use here. The amplitude of the quiver motion is approximately $0.025 \mathrm{~mm}$.

$\begin{array}{ll}\text { Radius of cylindrical waveguide } & 14 \mathrm{~mm} \\ \text { Length of waveguide } & 492.528 \mathrm{~mm} \\ \text { Input signal power } & 200 \mathrm{MW} \\ \text { Frequency of input signal, H01 wave } & 34.272 \mathrm{GHz} \\ \text { Wavelength of H01 wave } & 9.46 \mathrm{~mm} \\ \text { Beam radius } & 0.01 \mathrm{~mm} \\ \text { Beam current } & 1 \mathrm{~A} \\ \text { Displacement of-axis beam } & 6.47 \mathrm{~mm} \\ \text { Beam energy } & 6 \mathrm{MeV} \\ \text { Amplitude of magnetic intensity at the beam radius } & 45.3 \mathrm{kA} / \mathrm{m} \\ \text { Amplitude of magnetic induction at the beam radius } & 0.057 \mathrm{~T} \\ \text { Wiggler parameter K } & 0.05\end{array}$

Table I. Parameters for FEL-type undulations in a counter-propagating wave.

One result that we found is that there is no oscillatory instability using only the counterpropagating wave. A second result reveals the simple harmonic quiver motion of the electrons in the RF undulator fields, as shown in Fig. 7. We use a 2D CST Studio feature that provides a snapshot of the particle momentum and position in a narrow slice (e.g. $0.035 \mathrm{~ns}$ ) of the beam at a given location in the waveguide. A phase-space plot for the $\left(P_{\mathrm{x}}, x\right)$ data is shown in Fig. 7.

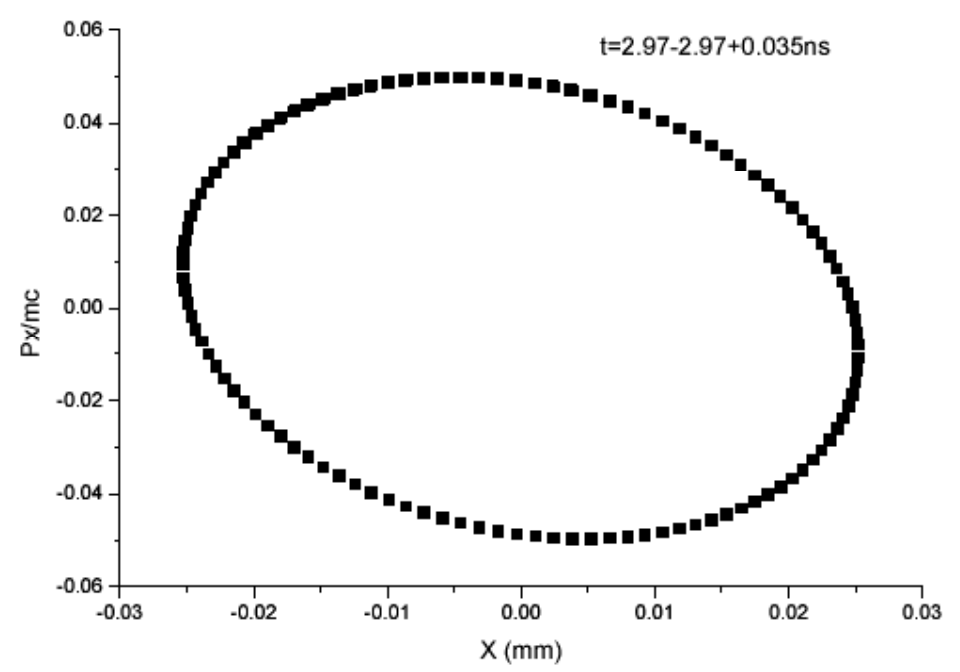

Fig. 7. Plot of the dynamical $\left(P_{\mathrm{x}}, x\right)$ coordinates of particles contained in a narrow slice of the beam at a given location ( $\mathrm{t}$ or $\mathrm{z}$ ) moving with energy $6 \mathrm{MeV}$ and under the influence of a counter-propagating wave rf undulator.

The closed elliptical pattern confirms oscillatory motion, the parameters of which agree with the FEL quiver motion estimates expected. The $\left(P_{\mathrm{y}}, x\right)$ values are found to be smaller by two orders of magnitude. The $\left(P_{\mathrm{x}}, x\right)$ data are uniform along $z$. 


\section{SHORT-PERIOD RF UNDULATOR FOR A SASE NANOMETER SOURCE}

Next, the counter-propagating wave was allowed to reflect and generate a co-propagating wave. When the small slice of particles was examined at a later time, both waves were present. Fig. 8 shows a very different result from Fig. 7. First, the amplitude of the motion has increased by two orders-of-magnitde. Secondly, the feature at the top right shows the particles are "spiraling" to larger amplitude during the sampling time. This is evidence of the instability that causes a coherent macroscopic oscillation of the beam, as shown in Fig. 5.

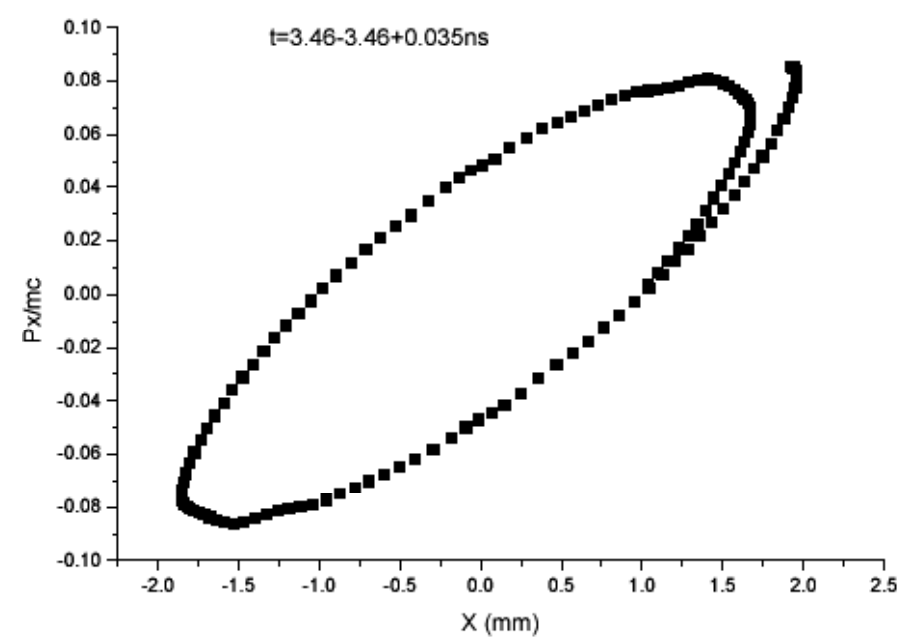

Fig. 8. Oscillatory motion of particles in presence of forward and backward waves.

In a further example, calculation of electron energies during the growing oscillatory instability set up by a co-propagating $\mathrm{TE}_{11}$ wave with an effective power of $400 \mathrm{MW}$ for a beam positioned at $r=0$ shows a large variation in particle energy of $\sim 100 \mathrm{keV}$, as seen in Fig. 9. Such a large energy variation would render FEL operation problematical, as the quality of the electron beam would very likely be compromised.

So, how can this "instability" be avoided while retaining the resonant cavity needed to build up high fields? The answer, devised by Omega-P senior research physicist S.V. Kuzikov, is to introduce mode converters at each end of the resonator. For illustration, suppose we position the beam at $r=0$, and provide a counter-propagating $\mathrm{TE}_{11}$ wave which has its maximum transverse fields on the axis. When this wave is reflected at the end of the resonator, imagine that it is converted to a $\mathrm{TE}_{01}$ wave having zero transverse field on axis; and when the $\mathrm{TE}_{01}$ wave is reflected from the opposite end of the resonator, it is converted back to the original $\mathrm{TE}_{11}$ mode, etc. (The large RF axial magnetic field will interact with the transverse quiver velocity set up by the FEL-driving counter-propagating wave to cause a second-order radial ponderomotive force 
Omega-P, Inc.

Final Report on 2010 SBIR Phase I grant DE-SC-0004491

\section{SHORT-PERIOD RF UNDULATOR FOR A SASE NANOMETER SOURCE}

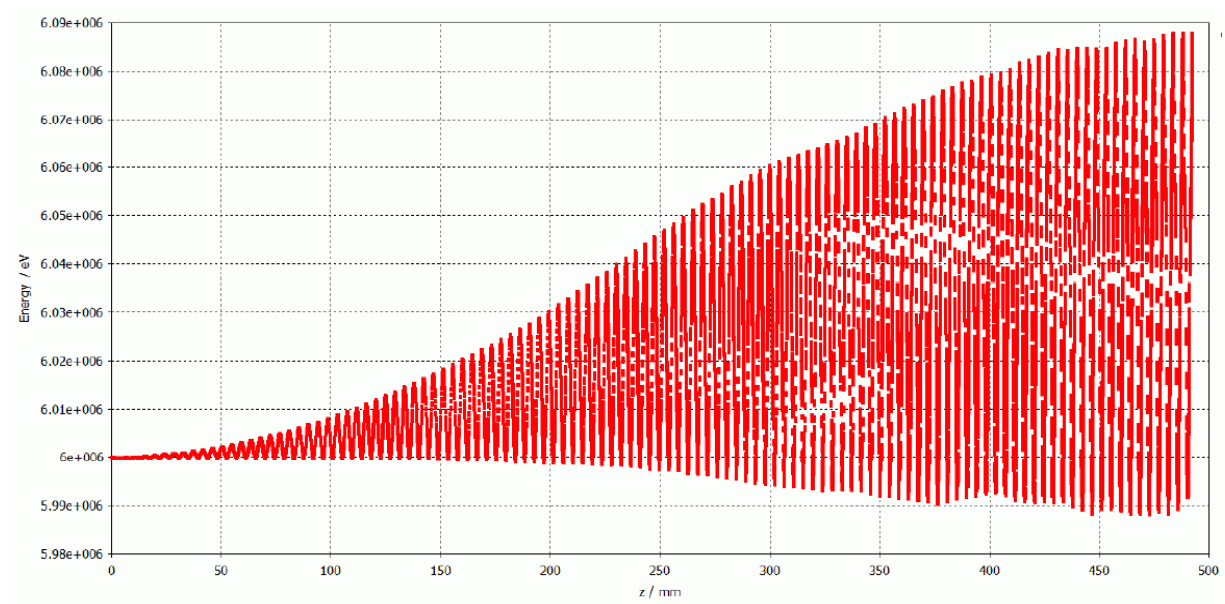

Fig. 9. Growing energy oscillations on a beam exposed to an intense co-propagating $\mathrm{TE}_{11}$ wave, corresponding to the spatial oscillations as seen as in Fig. 5.

that modulates the radius of the electron beam.) A section of the beam trajectories moving along the axis in the co-propagating $\mathrm{TE}_{01}$ wave is shown in Fig. 10a. The entire beam length of $50 \mathrm{~cm}$ is shown in Fig. 10b: no spatial instability is in evidence. Our conclusion is that using a copropagating $\mathrm{TE}_{01}$ wave with the beam on-axis will avoid the problem of the growing spatial instability.

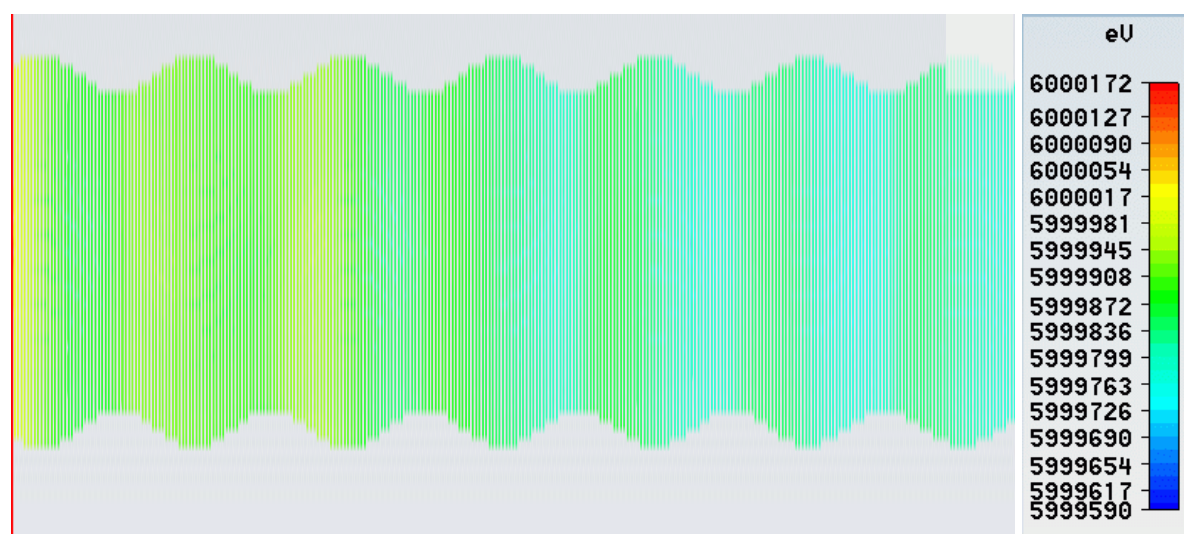

Fig. 10a. Radially-expanded view of electron beam in a $28.5 \mathrm{~mm}$ path after moving 2.2 $\mathrm{ns}$ in a forward wave. . Electrons travel from the right to the left. Space and energy scale periodicity is $4.4 \mathrm{~mm}$. Co-propagating wave (only), $\mathrm{TE}_{01}$ mode power is $400 \mathrm{MW}, K=$ 0.1 . The energy variation is $\sim 100 \mathrm{eV}$. 




Fig. 10b. Same as Fig. III-9a, but entire length of beam travel in $50 \mathrm{~cm}$ waveguide is shown. Note absence of large spatial instability in this case.

We now show results from the counter-propagating $\mathrm{TE}_{11}$ wave alone in Figs. 11a,b). These pictures show that the RF undulator is not distorting the overall beam motion.

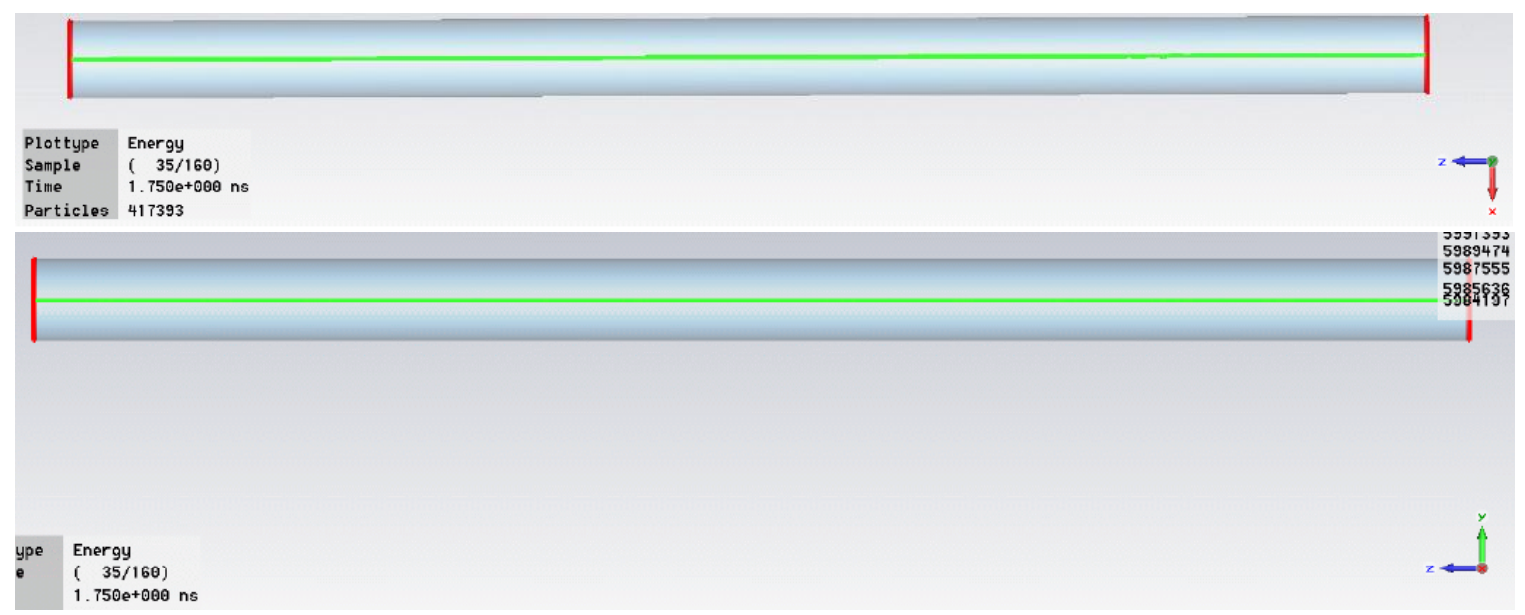

Fig. 11a (top, $x, z$ plane) and $-11 \mathrm{~b}$ (bottom, $y, z$ plane). Beam moves along the axis of a 14-mm radius cylindrical waveguide in a counter-propagating $\mathrm{TE}_{11}$ wave. $K=0.067$.

These results, which show how a RF undulator can induce unwanted and potentially harmful macroscopic motion of the beam (or bunch) are new and were not revealed in any previous work. Where does this effect come from? There has been a pattern among various researchers to recover the analogous FEL equations for the RF undulator and declare victory, without delving into the entire set of equations of motion to see what else may be involved. As a result, designs may contain unacceptable amounts of the co-propagating wave. We here present a short summary of our theory on this topic below. Shintake's [5] Eqn. 2.5, derived from the 
Omega-P, Inc.

Final Report on 2010 SBIR Phase I grant DE-SC-0004491

\section{SHORT-PERIOD RF UNDULATOR FOR A SASE NANOMETER SOURCE}

Lorentz force on a relativistic electron moving primarily along the $z$ axis in a transverse electric field of amplitude $E_{0}$, is of the form:

$$
\mathrm{d} \beta_{\mathrm{x}} / \mathrm{d} \tau=\mathrm{A} \cos (\tau)-\mathrm{A} \alpha \cos (\alpha \tau) \text {, where }: \tau=2 \pi z / \lambda_{\mathrm{u}}, \text { and where }
$$

$\mathrm{A}=e E_{0} \lambda_{0} / 2 \pi \gamma m c^{2}, \alpha=\left[\lambda_{\mathrm{g}}-\lambda_{0}\right] /\left[\lambda_{\mathrm{g}}+\lambda_{0}\right], \lambda_{\mathrm{u}}=\lambda_{\mathrm{g}} \lambda_{0} /\left[\lambda_{\mathrm{g}}+\lambda_{0}\right]$; with $\lambda_{\mathrm{u}}$ the undulator period.

The first term is due to the counter-propagating wave, while the second term is due to the copropagating wave. For a $\mathrm{TE}_{11}$ wave having frequency $34.3 \mathrm{GHz}$ and cutoff frequency $6.3 \mathrm{GHz}$, $\alpha=0.0085$. Taking $\beta_{\mathrm{x}}=0$ at $\mathrm{z}=0$, we find

$$
\beta_{\mathrm{x}}=\mathrm{A} \sin (\tau)-\mathrm{A} \sin (\alpha \tau)
$$

Thus, the deflection for the normalized coordinate $X=x\left(2 \pi / \lambda_{\mathrm{u}}\right)$ follows as:

$$
X=\mathrm{A}[1-\cos (\tau)]-(\mathrm{A} / \alpha)[1-\cos (\alpha \tau)]
$$

Where $X(0)=0$. If $\alpha<<1$, the second term (due to the co-propagating wave) dominates the first. From this it can be appreciated that whereas the co-propagating term can be dropped in Eq. (1), it cannot be dropped in Eqs. (2) or (3). [The radiation, depending on the acceleration given by Eq. (1), is mostly driven by the counter-propagating wave term.] The large additional term from the forward wave affects the particle motion in a manner that is very different from the magnetostatic undulator. In Fig. 12 we show a graph of the transverse motion of a test electron for the parameters cited above. It is seen that the scale of transverse motion is large compared with the FEL-type oscillations, as we have already found.
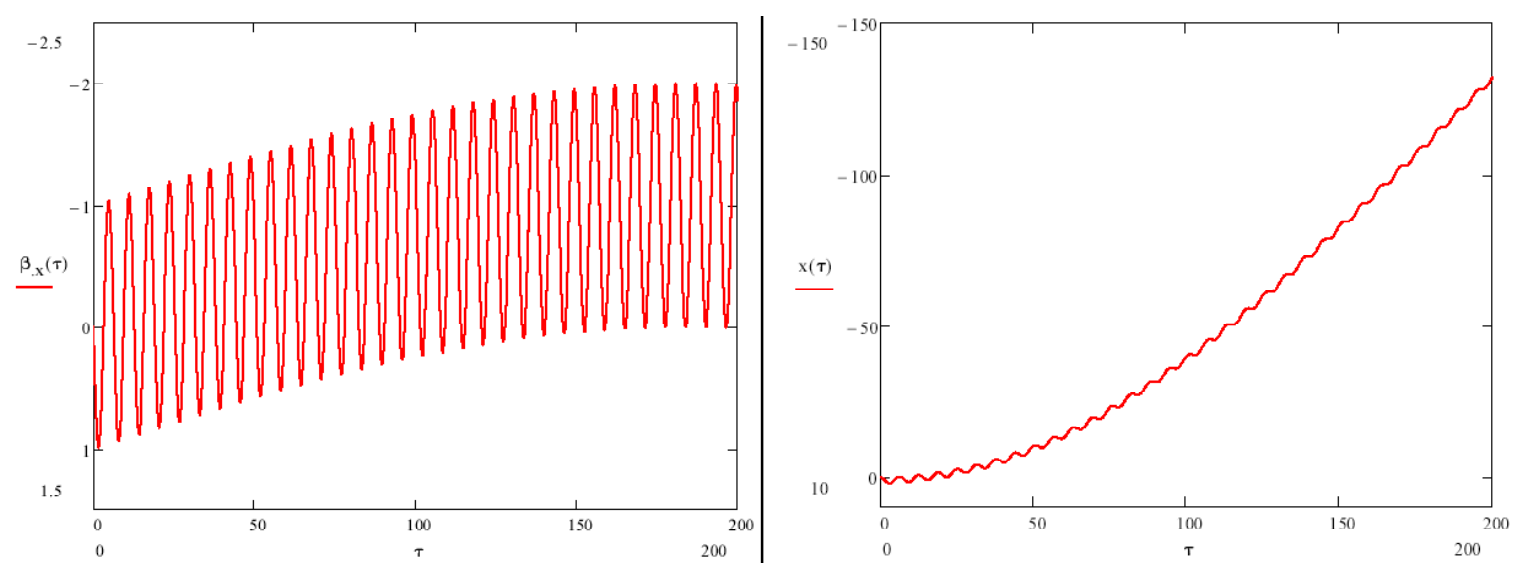

Fig. 12. Transverse motion of a test electron in co- and counter-propagating waves computed taking $A=1$ (arbitrary units: period and amplitude of FEL quiver motion provides a scale for comparison: see Fig. IIIb-6). (The transverse scale of $x$ is large here because the computation has taken a very large value of $K$ for this illustration.) 


\section{SHORT-PERIOD RF UNDULATOR FOR A SASE NANOMETER SOURCE}

The transverse motion is also affected to some degree by the ponderomotive force; this force $F_{\mathrm{y}}$ arises from the transverse quiver motion $v_{\mathrm{x}}$, via the term $v_{\mathrm{x}} B_{\mathrm{z}}$ in the Lorentz force equation. It should scale approximately as $K^{2}$, so for $K<1$ it need not be large and can be controlled by standard beam focusing techniques. The sign of this force (outward/inward) depends on the profile of $B_{\mathrm{z}}(x, y)$ where the charge is located. Stabilization can be provided, if called for, by conventional focusing techniques.

The prescription for a successful RF undulator obtained from this study is to use a counter-propagating $\mathrm{TE}_{11}$ wave (or equivalent) and a co-propagating $\mathrm{TE}_{01}$ wave (or equivalent), with in this case the beam positioned on the axis of symmetry. However, we have found that the $\mathrm{TE}_{11}$ mode could cause excessive wall losses and possibly high RF breakdown probability at the metallic surface: so the solution to both the wall problem and the backward/forward wave problem must involve a different choice of modes, as will be described in Section IId.

\section{IId. Microwave Undulator Resonator Designs}

In this section we describe results from our design studies of suitable microwave resonators for an RF undulator, in light of our newly-discovered need to avoid a co-propagating wave that can interact with the electron beam. As a result, the simple standing-wave cavity designs presented in the Phase I proposal need to be abandoned, and alternatives proposed. A number of alternative designs were studied, and two versions selected for presentation here. The first version is a resonator equipped with mode conversion reflectors, so that the counter- and copropagating modes are different (e.g., $\mathrm{TE}_{01}$ and $\mathrm{TE}_{02}$ modes of a cylindrical structure); and in which the electron beam moves parallel to but off the axis. The second is a resonant ring guiding a unidirectional $\mathrm{HE}_{11}$ mode that uses an impedance corrugation and oversize waveguide. The first of these (hereafter referred to as a two-mode resonator) is--to our knowledge--a totally new concept which obviously must be tested. The second is an extension of known technology [10], but will be shown to require much high power than the first; nevertheless, it still has practical advantages that will be discussed. As a result, our plan in Phase II would have been to refine the design for the two-mode resonator and evaluate a cold-test version. If at that stage, relatively early in the Phase II project, the two-mode resonator still seems promising, a high-power version would have been built. If, on the other hand, some unforeseen difficulty emerged, there would still have been time and resources to revert to the ring resonator, although the $K$-value that can be reached will probably be lower than might have been the case for the two-mode resonator, due to available RF power limitations.

\section{(1) $\mathrm{TE}_{01} / \mathrm{TE}_{02}$ cylindrical two-mode resonator}

Fig. 13 shows the resonator and its manner of excitation. It uses a $\mathrm{TE}_{01}$ counterpropagating wave and a $\mathrm{TE}_{02}$ co-propagating wave (other $\mathrm{TE}_{0 n}$ modes with $n>2$ do not propagate. The electron beam is located off-axis at the zero of the transverse magnetic field of the co-propagating $\mathrm{TE}_{02}$ mode; this position is close to the field maximum of the counter- 
Omega-P, Inc.

Final Report on 2010 SBIR Phase I grant DE-SC-0004491

\section{SHORT-PERIOD RF UNDULATOR FOR A SASE NANOMETER SOURCE}

propagating $\mathrm{TE}_{01}$ mode. These modes were described in the Phase I proposal as possessing a breakdown proof character (no $E$-fields at the wall), as well as to provide lower surface RF pulsed heating than some other modes.

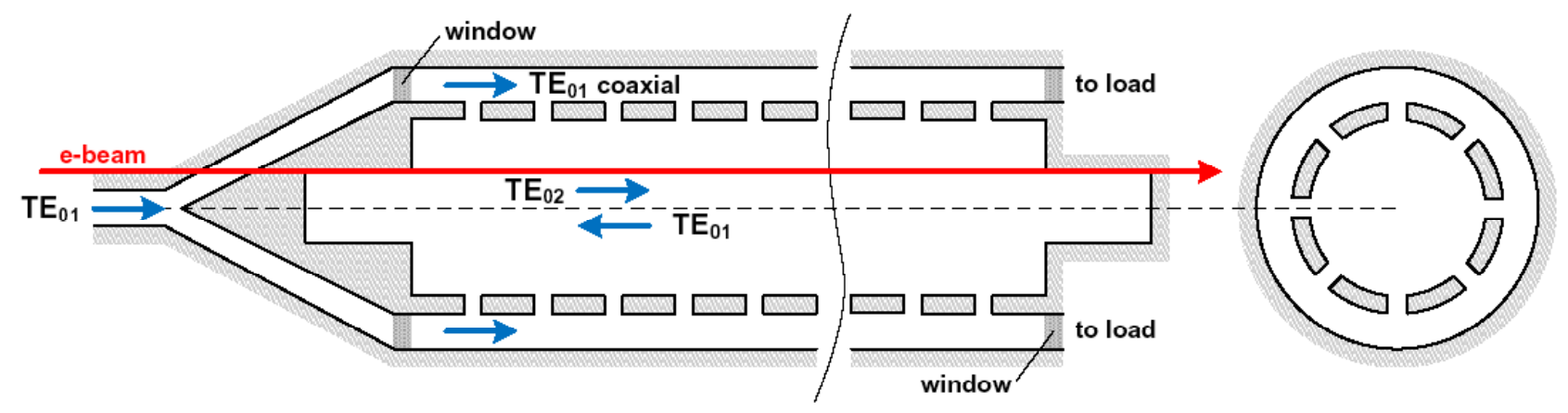

Fig. 13(a). Conceptual sketch of the two-mode resonator.
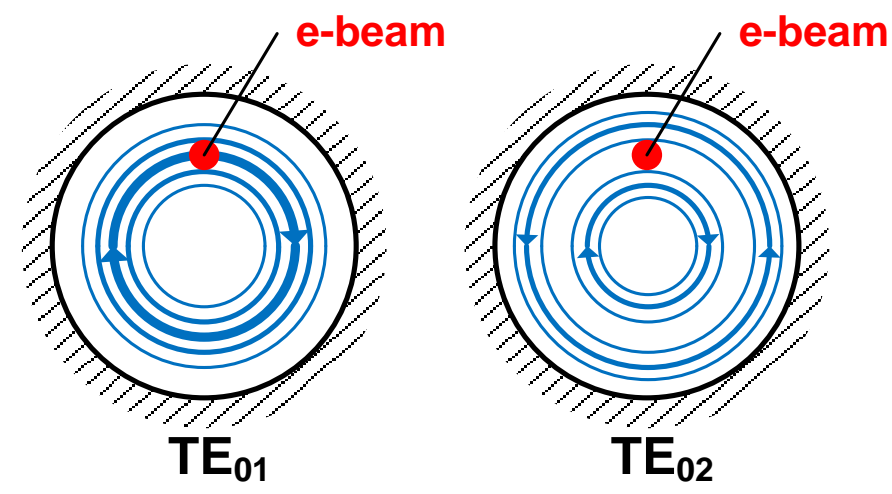

Fig. 13(b). Cross-section of the resonator showing azimuthal transverse electric fields of the two modes to be employed, and showing location of the beam.

The mode converter positioned at each end of the resonator consists of two connected waveguides having different radii, as shown in Fig. 14(a). The efficiency of conversion for this simple structure can be very high, with diffraction losses due to incomplete conversion between $\mathrm{TE}_{01}$ into $\mathrm{TE}_{02}$ (or vise-versa) $<0.001 \%$, as shown in Fig. 14(b). This design permits the use of mode filters inside the converter, using the principle of cutoff corrugation with absorbers. This latter concept is illustrated in Fig. 14(a).

The coupler uses a common perforated wall between the coaxial $\mathrm{TE}_{01}$ and $\mathrm{TE}_{02}$ modes in the main circular cross section waveguide (Fig. 15). The radius of the outer structure is 19.4 $\mathrm{mm}$, the periodicity of the holes is $3 \mathrm{~mm}$, the wall thickness is $2 \mathrm{~mm}$, the coupling hole diameter is $2.1 \mathrm{~mm}$, and there are 8 holes around the azimuth. The $\mathrm{TE}_{02}$ mode has the necessary $~ 1 \%$ coupling level, as seen in Fig. 16. All other unwanted modes have < $-40 \mathrm{db}$ excitation level. Table II shows operating parameters of this for a cavity operating at room temperature. 


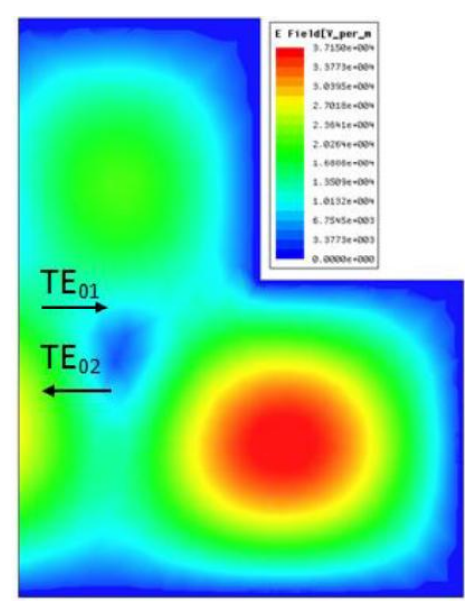

Fig. 14(a). Outline and electric field map for the $\mathrm{TE}_{01}$ to $\mathrm{TE}_{02}$ mode step-type mode converter. Axis is along bottom.

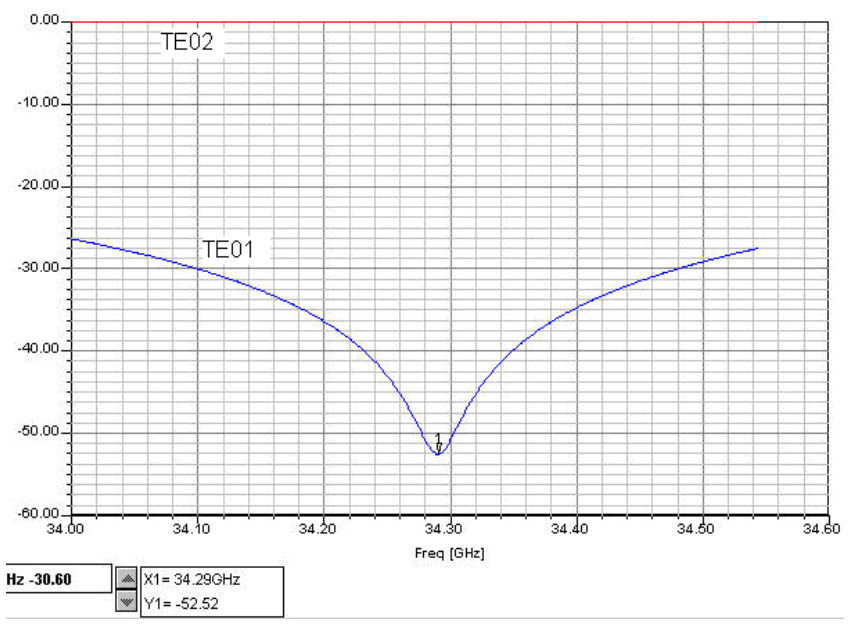

Fig. 14(b). Calculated example of reflected modes in the step-type mode converter.

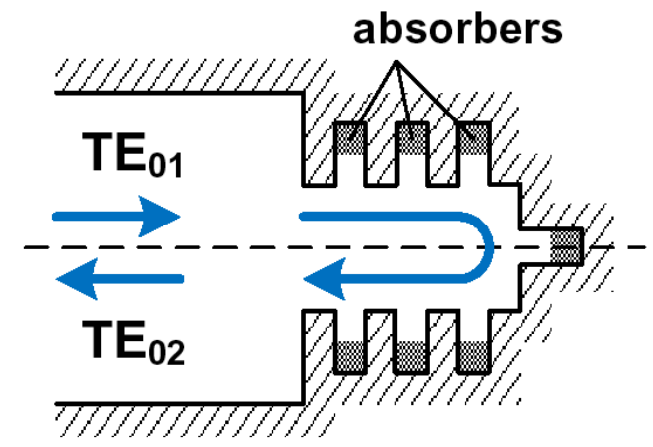

Fig. 14(c). $\mathrm{TE}_{01}-\mathrm{TE}_{02}$ mode step converter equipped with built-in mode filters.

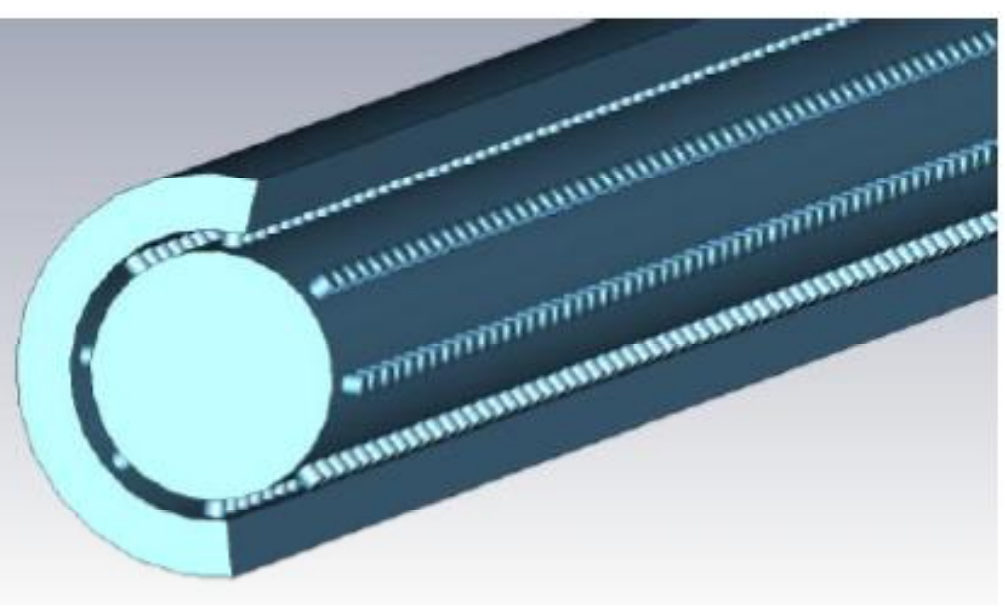

Fig. 15. Device showing the schematic of the input coupler. Light areas are open channels. 
Omega-P, Inc.

Final Report on 2010 SBIR Phase I grant DE-SC-0004491

\section{SHORT-PERIOD RF UNDULATOR FOR A SASE NANOMETER SOURCE}

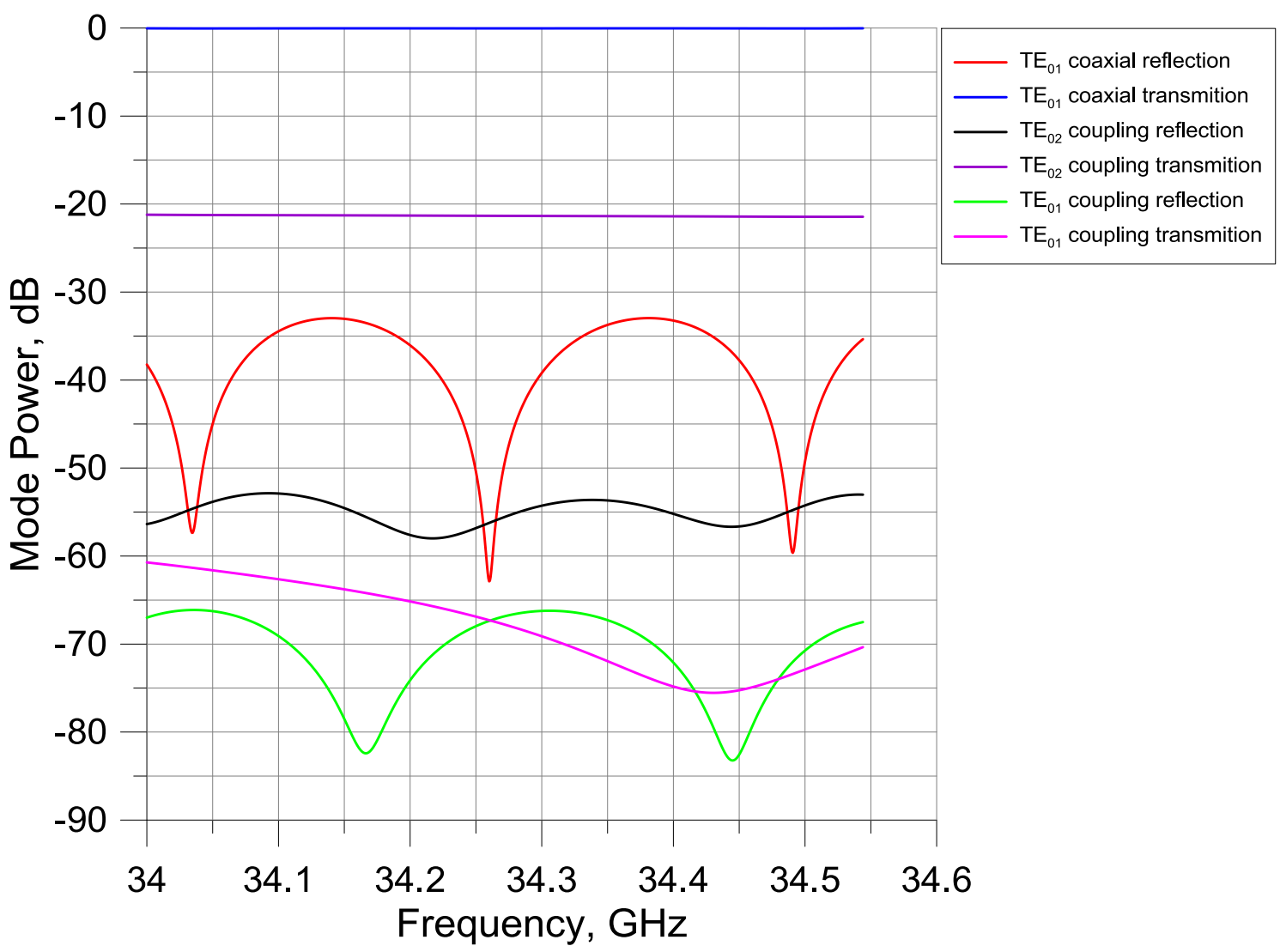

Fig. 16. Modes excited in the coaxial input coupler for the two-mode cavity.

Table II. Parameters for the two-mode cavity.

Note some finite surface field is expected at the coupling holes.

\begin{tabular}{|c|c|c|}
\hline main section radius, $R$ & 12 & $\mathrm{~mm}$ \\
\hline main section length, $L$ & 500 & $\mathrm{~mm}$ \\
\hline $\mathrm{TE}_{01}$ group velocity, $v_{\mathrm{gr}} / c$ & 0.895 & \\
\hline $\mathrm{TE}_{02}$ group velocity, $v_{\mathrm{gr}} / c$ & 0.580 & \\
\hline Ohmic quality factor, $Q_{\mathrm{ohm}}$ & 13,6000 & \\
\hline loaded quality factor, $Q_{\mathrm{L}}$ & 6,8000 & $\%$ \\
\hline coupling, $1-r^{2}$ & 0.8 & $\mathrm{~ns}$ \\
\hline $\mathrm{RF}$ pulse duration, $\tau$ & 630 & $\mathrm{~mm}$ \\
\hline beam shift off axis, $r_{\mathrm{b}}$ & 6.55 & $\mathrm{MW}$ \\
\hline undulator parameter, $K$ & 0.4 & $\mathrm{MV} / \mathrm{m}$ \\
\hline peak RF input power, $P_{\mathrm{in}}$ & 34 & \\
\hline surface electric field $E_{\mathrm{s}}$ & 0 & \\
\hline
\end{tabular}

This structure has a major advantage of having zero surface electric field (and thus low Ohmic and thermal losses), and it filled with energy using a reasonable fraction of the pulse length of the magnicon. The power level required to reach $K=0.4$ is beyond the present 
Omega-P, Inc.

Final Report on 2010 SBIR Phase I grant DE-SC-0004491

\section{SHORT-PERIOD RF UNDULATOR FOR A SASE NANOMETER SOURCE}

delivered capability of our magnicon by a factor about four-to-one, but it still would provide us with a laboratory test with $K \sim 0.2$, that should have been adequate for our Phase II experimental work. Moreover, a re-build of the magnicon is scheduled for 2012--subject to availability of funds--that would bring the output power level to $>20 \mathrm{MW}$.

It can also be pointed out that the use of liquid nitrogen cooling will lower the required RF power from $34 \mathrm{MW}$ to $13 \mathrm{MW}$, but we do not necessarily plan to use this method to confirm the device performance. It should be noted that the power needed is high because we have chosen a device that is $50 \mathrm{~cm}$ in length, longer than the structures contemplated in the Phase I proposal; thus, to increase $K$ for our planned studies at Yale that use lower power from the magnicon, we can also consider shortening this structure. It should also be noted that since this structure has cylindrical symmetry it would only produce linearly-polarized SASE radiation.

\section{(2) RF Undulator Using a $\mathrm{HE}_{11}$ Resonant Ring}

As stated above, a resonant ring seems to be an attractive candidate for a RF undulator, since it supports a guided wave travelling in only one direction--here the counter-propagating wave. Omega-P has had considerable experience with a mm-wave resonant ring [10], having designed and tested (with Institute of Applied Physics) the device shown in Figs. 17 and 18.

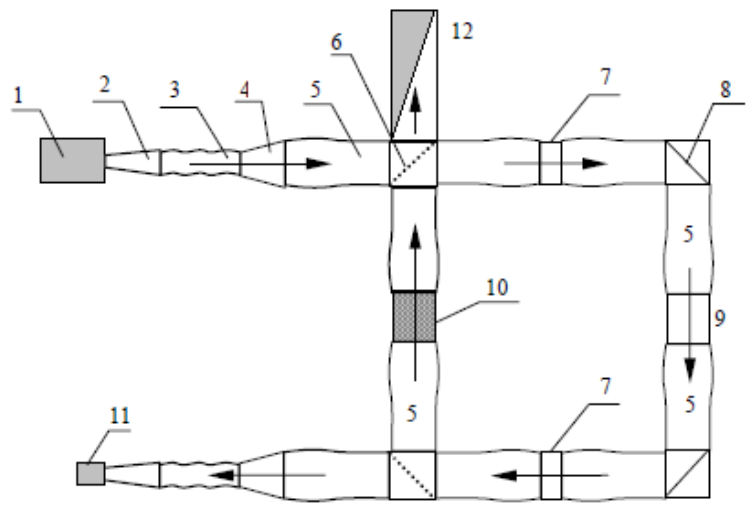

Figure 4. Schematic of overmoded Ka-band high-power resonant ring.

1 - microwave source;

$2-\mathrm{TE}_{10} \Rightarrow \mathrm{TE}_{11}$ mode converter;

$3-\mathrm{TE}_{11} \Rightarrow \mathrm{TE}_{01}$ mode converter;

$4-\mathrm{TE}_{01}$ waveguide up-taper;

$5-\mathrm{TE}_{01} \Rightarrow \mathrm{TE}_{01}+\mathrm{TE}_{02}$ mode converters:

6 - coupling mirror;

7 - bellows for frequency adjustment;

8 - flat mirror miter bends;

$9-\mathrm{TE}_{01}$ circular waveguide;

10 - component under test;

11 - microwave detector,

12 - microwave load.

Fig. 17. Details of the IAP/Omega-P Ka-band resonant ring [10]. 


\section{SHORT-PERIOD RF UNDULATOR FOR A SASE NANOMETER SOURCE}

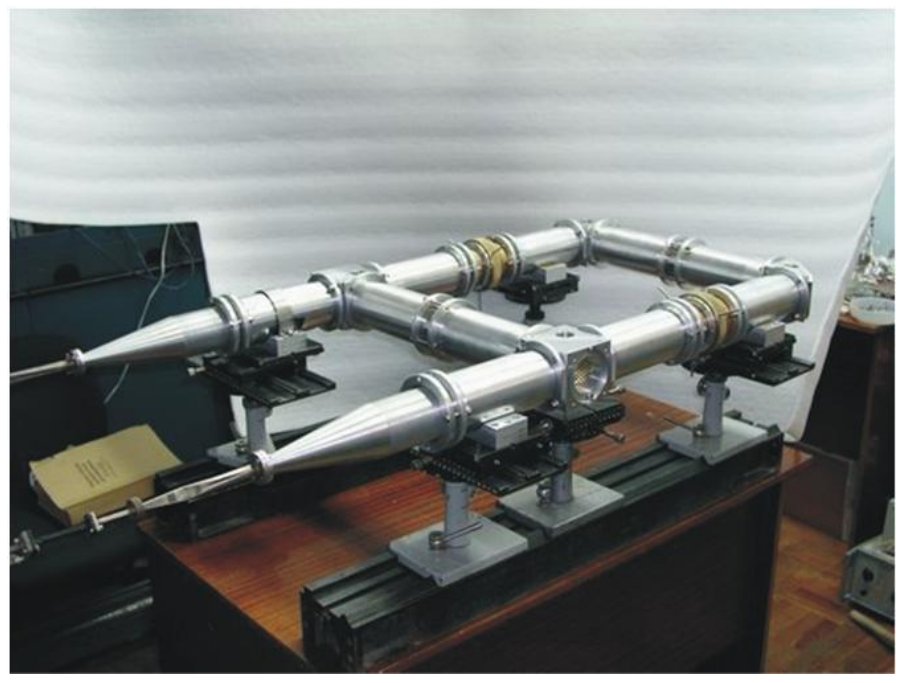

Fig. 18. Photograph of the IAP/Omega-P Ka-band resonant ring [10].

This resonant ring, operating in the $\mathrm{TE}_{01}$ mode, exhibited an effective RF power gain of 35:1.

A ring structure for the RF undulator has been designed, using for the counter-propagating waves a combination of the $\mathrm{HE}_{11}$ and $\mathrm{HE}_{12}$ modes, as shown in Figs. 19 and 20. The ring uses an oversize waveguide with impedance corrugations; the period of corrugation is $\leq \lambda / 2$, and the depth of corrugation is $\approx \lambda / 4$. The corrugation allows a marked reduction in Ohmic losses. The diameter of the waveguide is much larger than the wavelength, as is necessary in order to provide low diffraction losses in the miter bends; the same requirement motivates an admixture of $\sim 25 \% \mathrm{HE}_{12}$ mode. There are two beat periods of the $\mathrm{HE}_{11}$ and $\mathrm{HE}_{12}$ modes in the $50 \mathrm{~cm}$ arm containing the beam. The lateral arms are much shorter, to reduce as much as possible the stored RF energy.

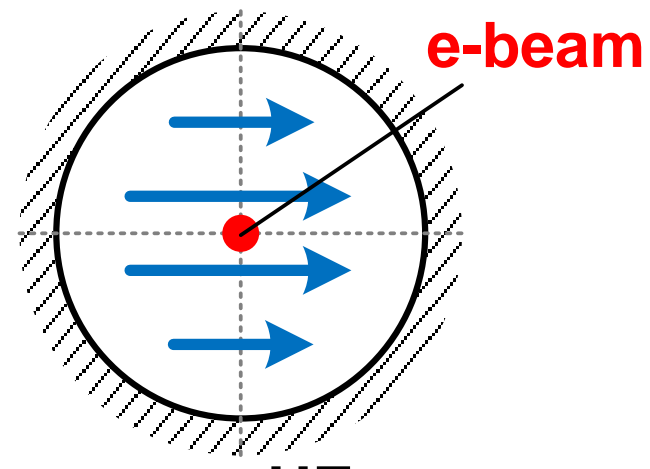

$\mathrm{HE}_{11}$

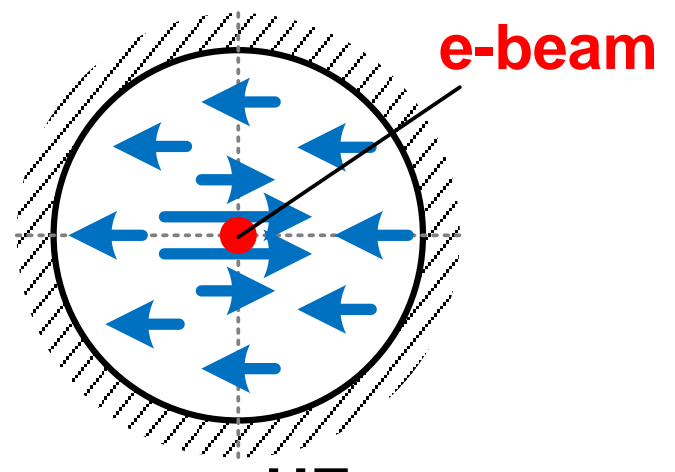

$\mathrm{HE}_{12}$

Fig. 19. RF magnetic field patterns and electron beam location in the resonant ring. 
Omega-P, Inc.

Final Report on 2010 SBIR Phase I grant DE-SC-0004491

SHORT-PERIOD RF UNDULATOR FOR A SASE NANOMETER SOURCE

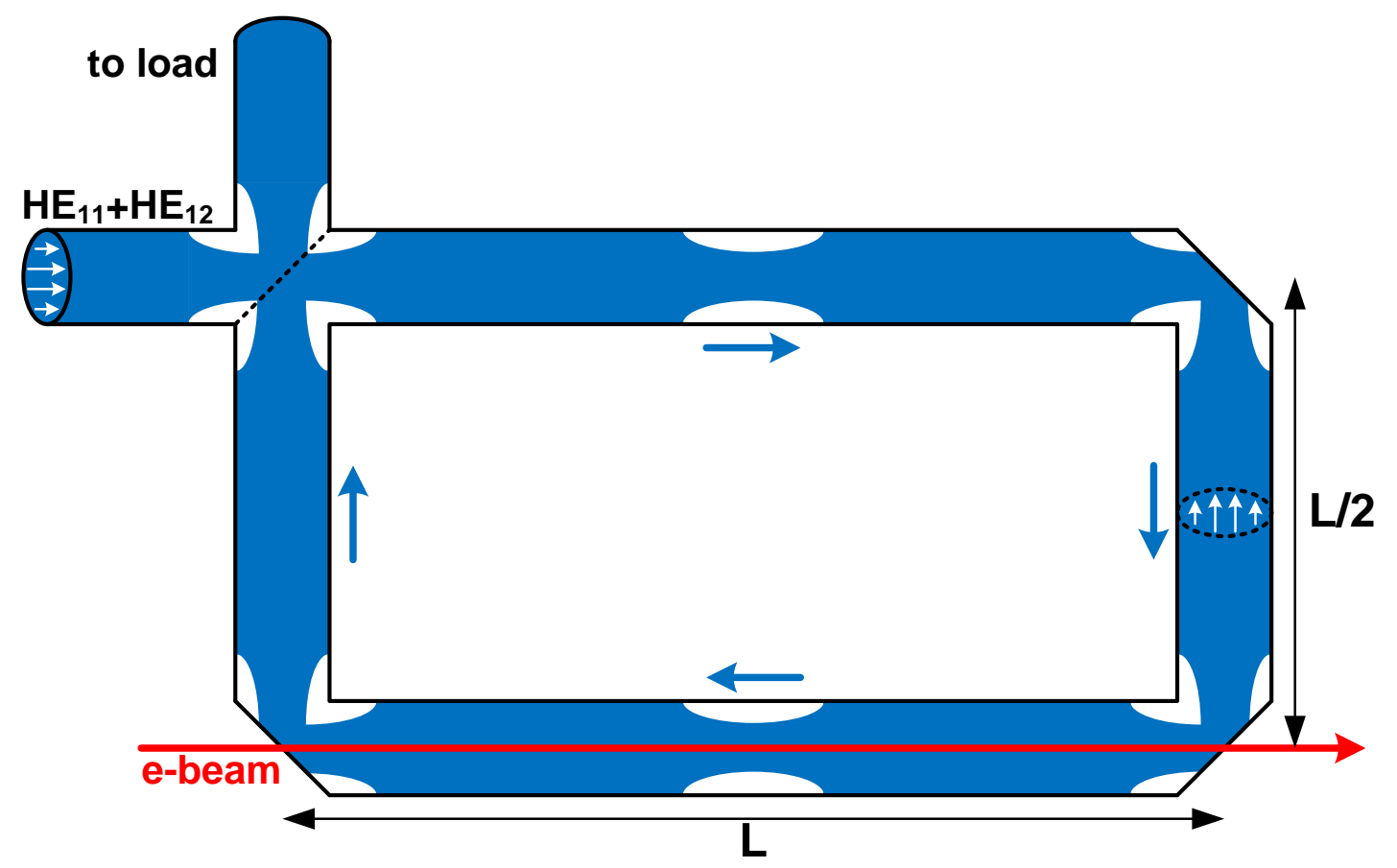

Fig. 20. Layout of the ring resonator layout, showing mode beat patterns (in blue), miter bends, input coupler, and location of electron beam..

The input coupler, sketched In Fig. 21, is actually a diffraction grating that provides coupling at the desired level of $1 \%$. The spacing of holes is small enough so as not to allow any other diffraction maxima except reflection and transmission. For numerical optimization we used a model shown in Fig. 22, where the plane wave of the finite aperture in rectangular waveguide illuminates a piece of the grating contained 3three holes. The optimized parameters that provides necessary coupling are $D=3.5 \mathrm{~mm}, \mathrm{~d}=2.2 \mathrm{~mm}, \Delta=1 \mathrm{~mm}$ (Fig. 23).

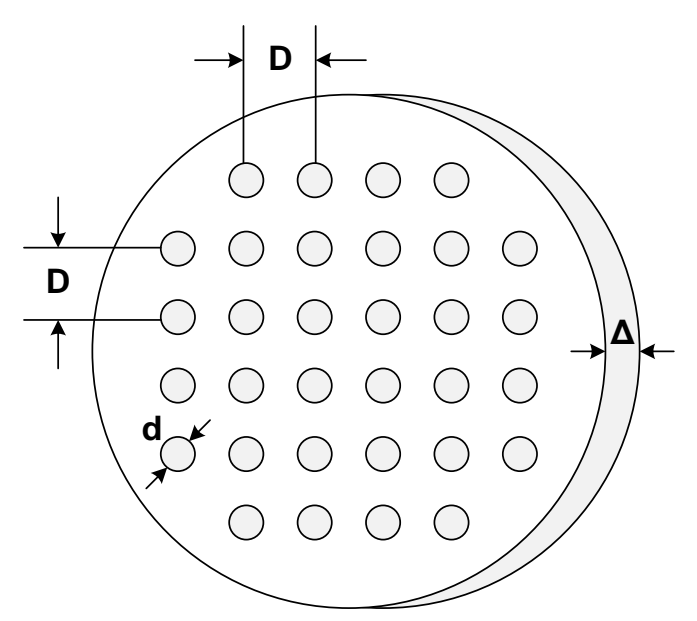

Fig. 21. Partially transparent diffraction grating to feed $\mathrm{HE}_{11}$ mode resonant ring. 
Omega-P, Inc.

Final Report on 2010 SBIR Phase I grant DE-SC-0004491

\section{SHORT-PERIOD RF UNDULATOR FOR A SASE NANOMETER SOURCE}

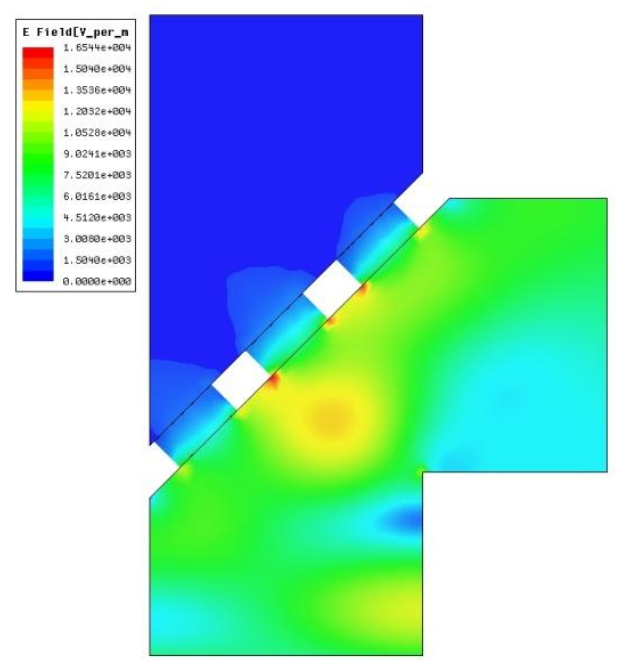

Fig. 22. Simulation model for coupler.

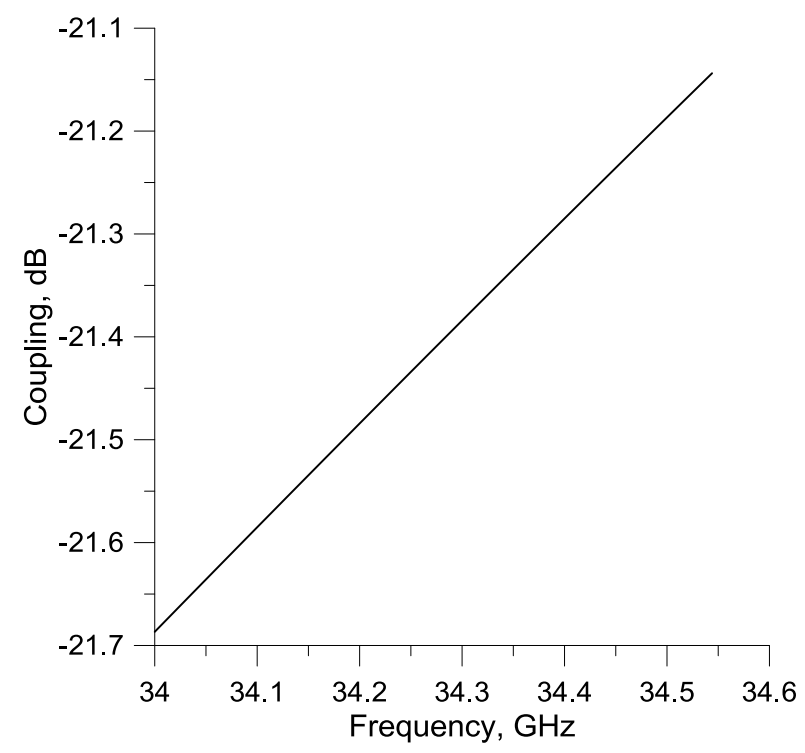

Fig. 23. Computed coupling factor provided by diffraction grating.

The concept for low loss miter bends is based on beating an admixture of $75 \% \mathrm{HE}_{11}$ and $25 \% \mathrm{HE}_{12}$ modes, as shown in Fig. 24. Field distribution of combined modes is shown in Fig. 25. At the entrance to a miter bend at cross-section 1 in Fig. 25, mutual phases of the operating modes corresponds to converging wave flow. (See Fig. 13 a,b). Such a wave has a waist (plane phase front) exactly in the center of the reflecting mirror. Here the wave-beam transverse size is smallest, so that the wave-beam is not perturbed by scattering at the edges. 
Omega-P, Inc.

Final Report on 2010 SBIR Phase I grant DE-SC-0004491

SHORT-PERIOD RF UNDULATOR FOR A SASE NANOMETER SOURCE

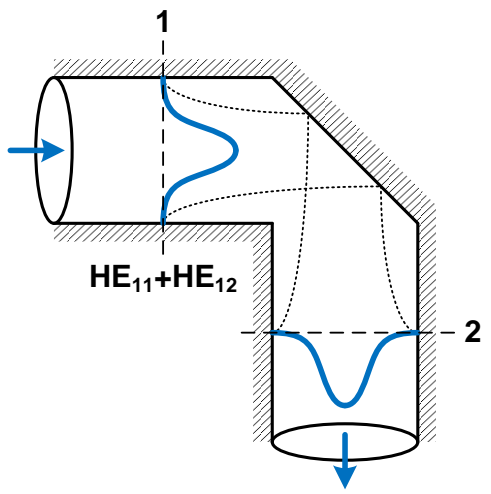

Fig. 24. Model for computation of miter bends.

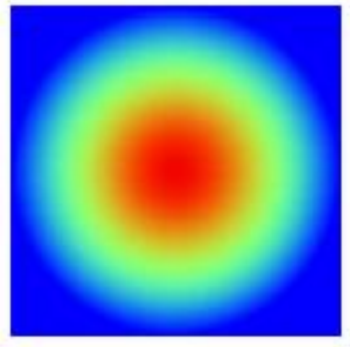

(a)
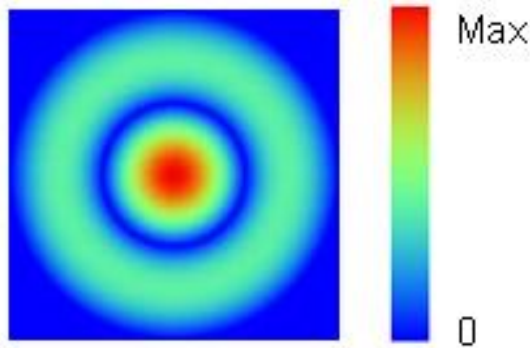

(b)

Fig. 25. Field distribution of operating modes:: (a) $-\mathrm{HE}_{11}$, (b) $-\mathrm{HE}_{12}$.

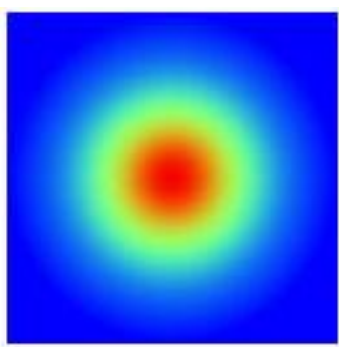

(a)

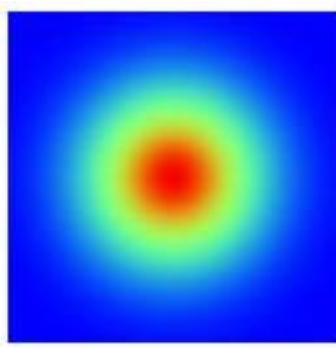

(c)

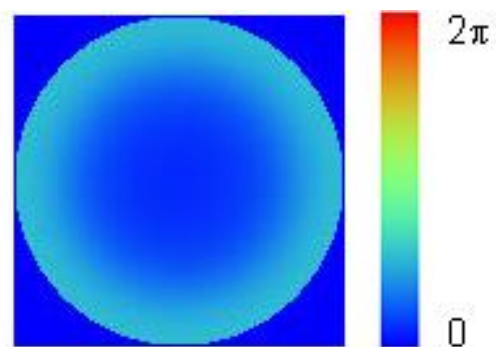

(b)

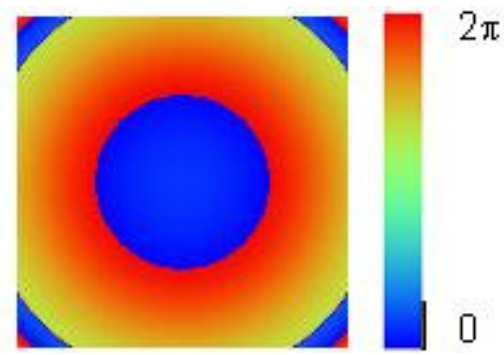

(d)

Fig. 26. Field (a) and phase (b) distributions of $\mathrm{HE}_{11}+\mathrm{HE}_{12}$ mode superposition at the entrance of miter bend, and field (c) and phase (d) for the resulting distributions at exit of miter bend.

[Note phase flip of $2 \pi$ in $(d)$, equivalent to constant phase across the aperture.] 


\section{SHORT-PERIOD RF UNDULATOR FOR A SASE NANOMETER SOURCE}

At cross-section 2, mutual phase of the operating modes takes the opposite sign. This is to correspond a wave-beam of the same amplitude distribution as the beam in cross-section 1, but a new phase distribution is actually the conjugated phase as regards to cross-section 1 (Fig. 26 c,d). Diffraction loss for a waveguide of radius $R=26.5 \mathrm{~mm}$ is $0.12 \%$. These losses grow if one reduces waveguide diameter. That is why an $\mathrm{HE}_{11}$ resonant ring inevitably has big volume and as a consequence requires relatively high RF power and stored energy. Miter bends can be easily designed with mode filters based on using absorbers at top and at bottom of the waveguide connection to improve mode purity.

At high power levels the impedance corrugation can lead to field enhancement levels that might lead to breakdown. Thus we have analyzed field magnitudes in the corrugation. In Fig. 27, the field distribution of the $\mathrm{HE}_{11}$ mode in corrugated waveguide is shown. The incident wave consists of $85 \% \mathrm{TE}_{11}$ mode and $15 \% \mathrm{TM}_{11}$ mode in a smooth circular waveguide. (This mode superposition provides a near-Gaussian field distribution--close to the desired distribution of the $\mathrm{HE}_{11}$ mode). One can see that fields in the corrugation are rather small even with enhancement caused by small radii taken into account. The surface $E$-field does not exceed $10 \%$ of $E$ at its maximum in the waveguide center, where $E$ equals approximately $160 \mathrm{MV} / \mathrm{m}$.

It should be noted that the $\mathrm{HE}_{11}$ and $\mathrm{HE}_{12}$ modes have small longitudinal $E_{\mathrm{z}}$ and $H_{\mathrm{z}}$ components. Both are less than the main $E_{\mathrm{x}}$ and $H_{\mathrm{y}}$ components by a factor of $1 /(k R)$. These components are zero in the center of the waveguide (at $r=0$ ). However, near the miter bends the total field consists not only of counter-propagating $\mathrm{HE}_{11}$ and $\mathrm{HE}_{12}$ modes, but also waves reflected with phase shifts of $90^{\circ}$, which can perturb the electron beam. However, in a final RF undulator using a resonant ring 10's of meters long there are need only be two miter bends encountered by the beam, as shown in the sketch in Fig. 28.

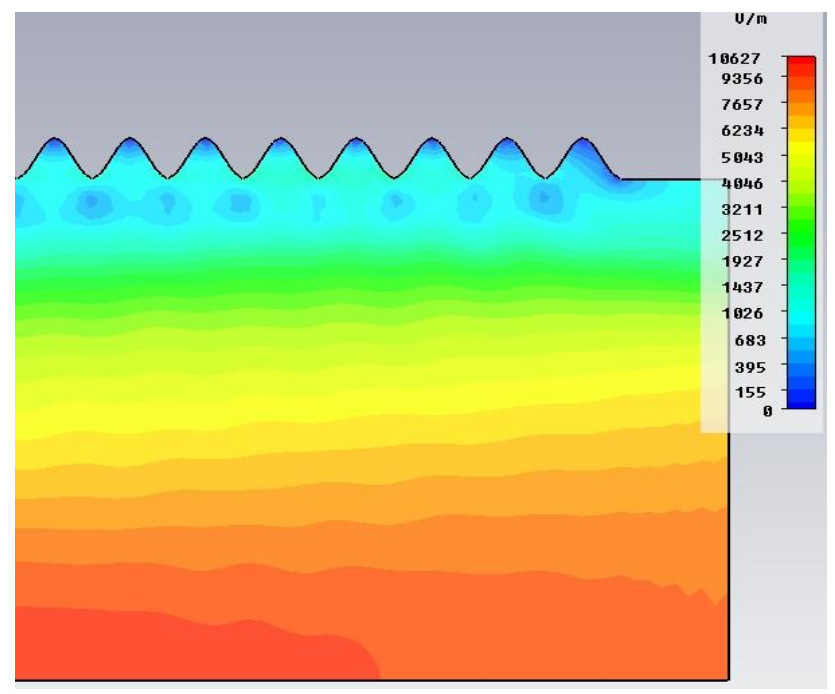

Fig. 27. Field distributions of the $\mathrm{HE}_{11}$ mode near the wall corrugations. 
Omega-P, Inc.

Final Report on 2010 SBIR Phase I grant DE-SC-0004491

\section{SHORT-PERIOD RF UNDULATOR FOR A SASE NANOMETER SOURCE}

A summary of the performance of the ring operating parameters at room temperature is provided in Table III. Although the surface field is acceptable at the corrugation, at the miter bends it is $\sim 220 \mathrm{MV} / \mathrm{m}$ (in one of polarizations); also the high level of power required to drive the ring resonator does not allow to consider this design without alternatives.

Table III. Parameters for a resonant ring RF undulator.

\begin{tabular}{|c|c|c|}
\hline waveguide radius, $R$ & 26.5 & $\mathrm{~mm}$ \\
\hline main section length, $L$ & 500 & $\mathrm{~mm}$ \\
\hline total length, $L_{\text {tot }}$ & 1500 & $\mathrm{~mm}$ \\
\hline $\mathrm{HE}_{11}$ group velocity, $v_{\mathrm{gg}} / c$ & 0.991 & \\
\hline $\mathrm{HE}_{12}$ group velocity, $v_{\mathrm{gr}} / c$ & 0.957 & \\
\hline Ohmic quality factor, $Q_{\mathrm{ohm}}$ & 43,6000 & \\
\hline diffraction quality factor, $Q_{\mathrm{Diffr}}$ & 22,8000 & \\
\hline loaded quality factor, $Q_{\mathrm{L}}$ & 7,5000 & \\
\hline coupling, $1-r^{2}$ & 0.73 & $\%$ \\
\hline pulse duration, $\tau$ & 700 & $\mathrm{~ns}$ \\
\hline beam axis shift, $r_{\mathrm{b}}$ & 0 & $\mathrm{~mm}$ \\
\hline undulator parameter, $K$ & 0.4 & \\
\hline input power, $P_{\mathrm{in}}$ & 120 & $\mathrm{MW}$ \\
\hline surface electric field $E_{\mathrm{s}}$ & 20 & $\mathrm{MV} / \mathrm{m}$ \\
\hline
\end{tabular}

The resonant ring concept can be expanded to the full length of RF undulator, consisting of several modules fed by several RF sources. As shown in Fig. IIIc-14, the electron beam propagates in a straight waveguide section, while the upper waveguide can have several bends in order to allow feeding by individual RF sources. The length of the wiggling sectio is determined by longest available pulse length of the sources.

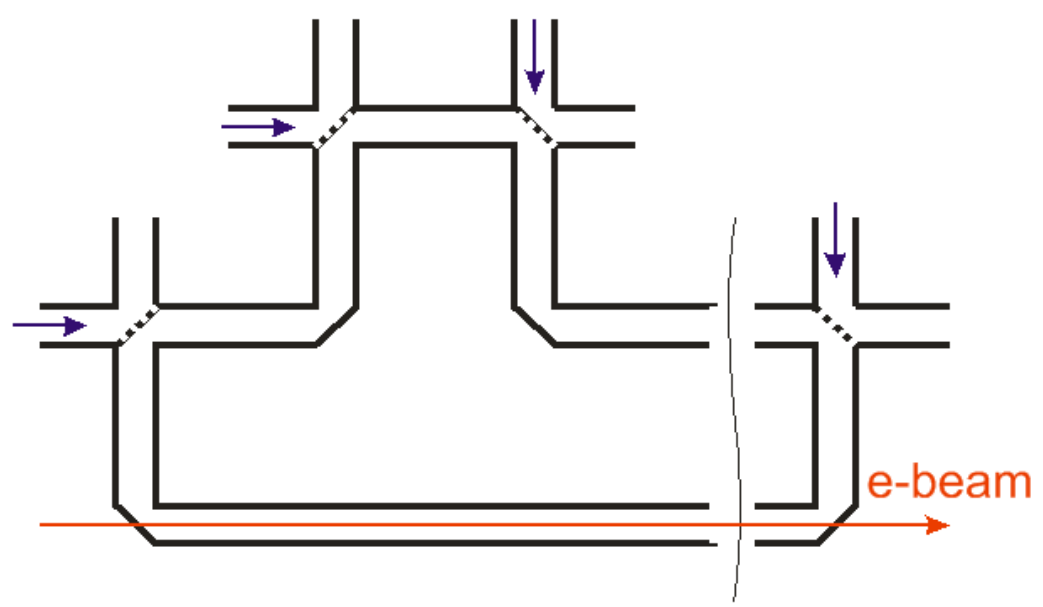

Fig. 28. Scheme of a long RF undulator consisting of several modules and fed by several RF sources. 
Omega-P, Inc.

Final Report on 2010 SBIR Phase I grant DE-SC-0004491

\section{SHORT-PERIOD RF UNDULATOR FOR A SASE NANOMETER SOURCE}

Analysis of these designs has included a study of techniques to reduce unwanted modes to the minimum levels as required by the analysis of Section IIb. In the following sub-section, we discuss tolerance studies for the designs.

\section{(3) Tolerance studies}

The most critical issue of the $\mathrm{TE}_{01}-\mathrm{TE}_{02}$ resonator is, of course, the mode converters, which must provide very high efficiency of conversion as it was shown in the previous section. Thus it is critical to investigate the levels of mechanical tolerance that can be allowed before an undue loss on mode-to-mode conversion efficiency results. The parameters of simulations used for studying tolerence are shown in Fig. 29. The results of this analysis are presented in Figs. 30-33 predict rather firm conclusion that dimensional accuracy of mode converter sizes should not be worse than several tens of microns. Green curves in the mentioned figures shows acceptable diffraction loss level which is necessary in order to avoid excitation of the spurious degenerate mode. Among typical imperfections of the real resonator one should also consider possible inclination of a mode converter relative axis of the resonator. Results of simulations for this particular case are plotted in Fig. 33. As one can conclude the acceptable inclination range is approximately $\pm 1^{\circ}$ in order to keep the additional losses to be less than other already existing losses, such as Ohmic losses.

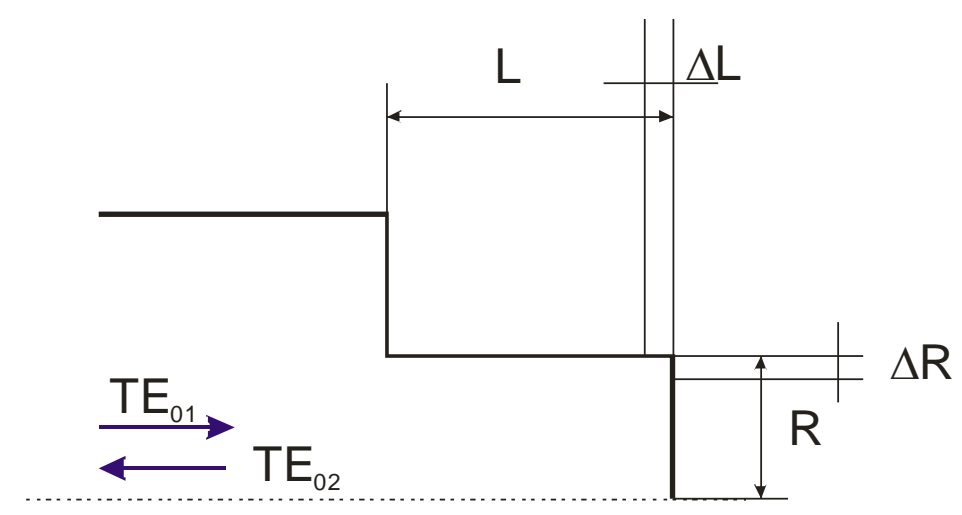

Fig. 29. Parameters for investigation of tolerance in $\mathrm{TE}_{01}-\mathrm{TE}_{02}$ mode converter. 
Omega-P, Inc.

Final Report on 2010 SBIR Phase I grant DE-SC-0004491

SHORT-PERIOD RF UNDULATOR FOR A SASE NANOMETER SOURCE

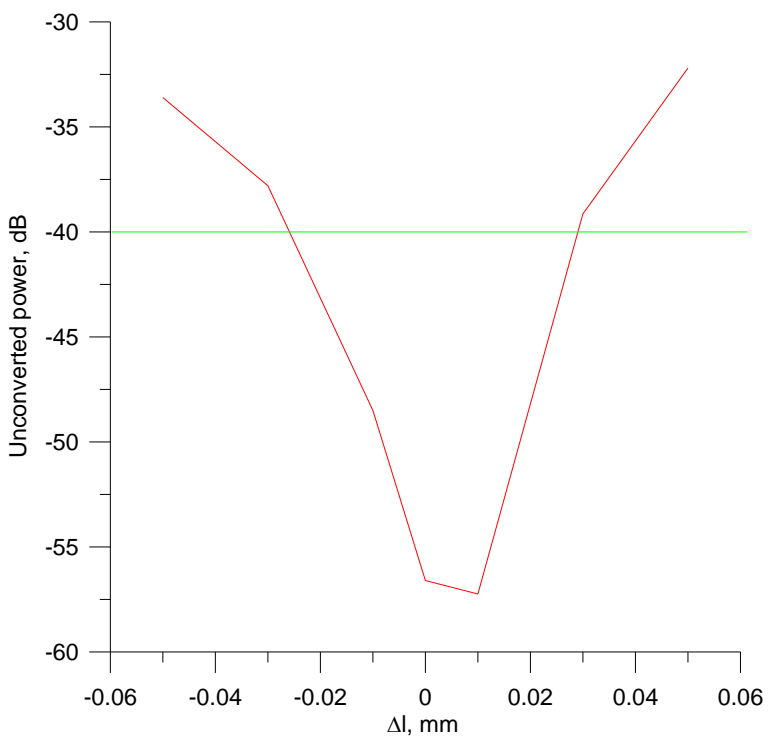

Fig. 30. Diffraction losses due to incomplete conversion of $\mathrm{TE}_{01}$ mode into $\mathrm{TE}_{02}$ mode caused by length error. Green curve shows acceptable level.

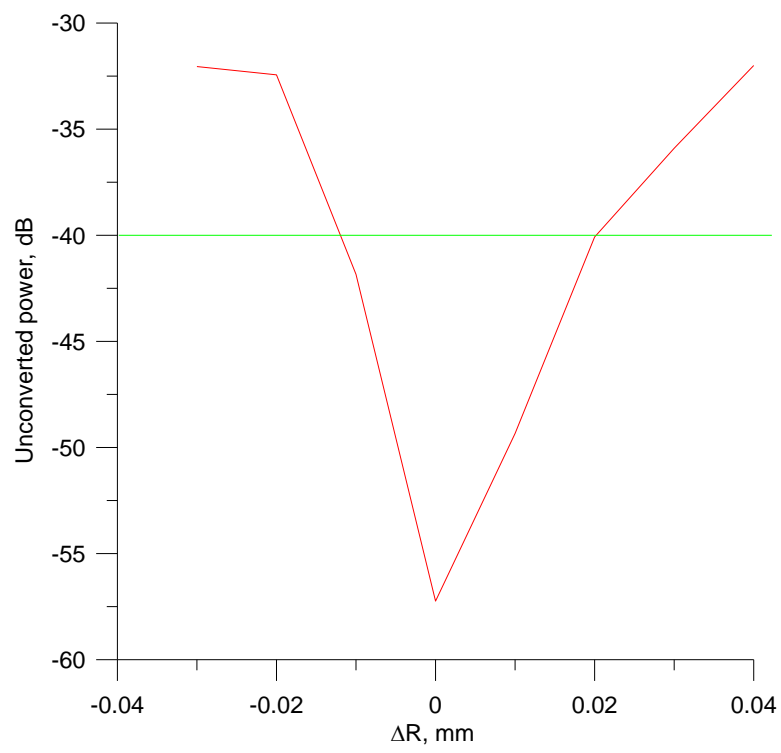

Fig. 31. Diffraction losses due to incomplete conversion of $\mathrm{TE}_{01}$ mode into $\mathrm{TE}_{02}$ mode caused by radius error. Green curve shows acceptable level.

Tolerance analysis of the $\mathrm{HE}_{11}-\mathrm{HE}_{12}$ resonant ring also included studies of unintended inclination of the mode converter and of the mirror in a miter bend, with respect to the resonator axis, with results shown in Fig. IIIc-21 and IIIc-22. Because inclination has a greater influence

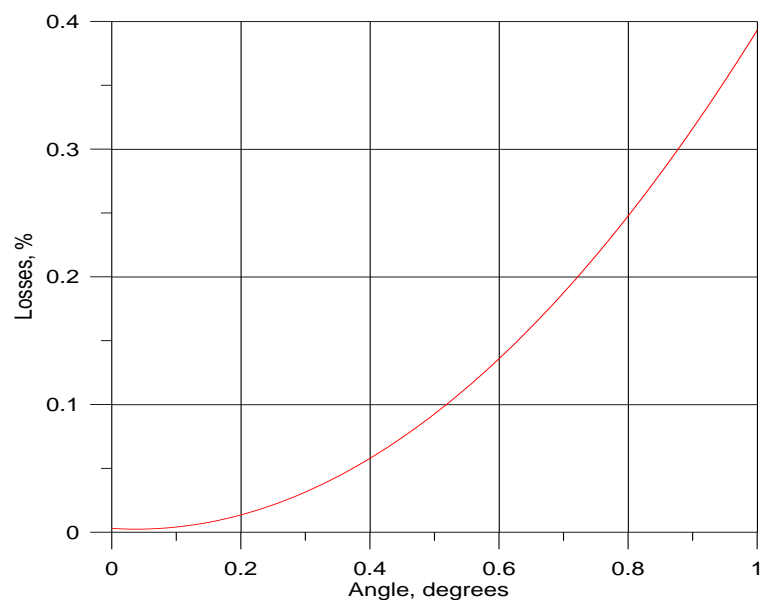

Fig. 32. Additional diffraction losses in $\mathrm{TE}_{01}-\mathrm{TE}_{02}$ mode converter caused by inclination of mode converter. Total losses (Ohmic and diffraction) without any inclination are $\sim 1 \%$.

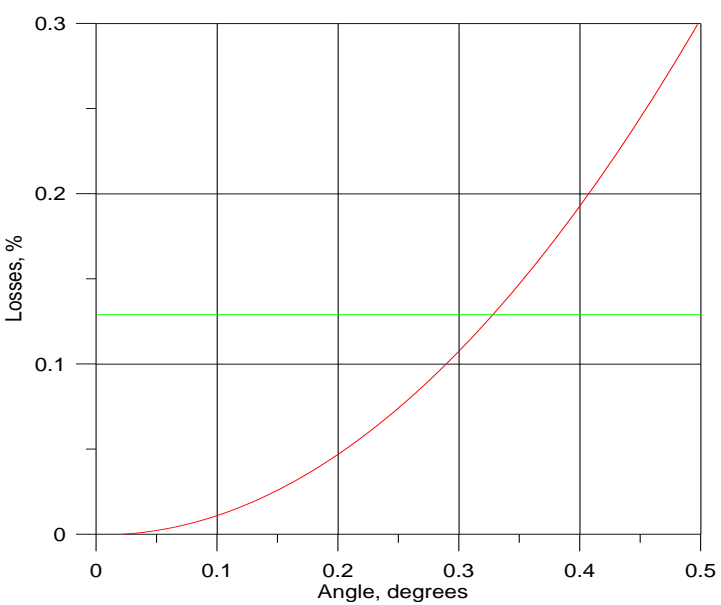

Fig. 33. Additional diffraction losses in resonant ring caused by inclination of miter bend mirror. Green line shows diffraction losses in ideal miter bend. 
Omega-P, Inc.

Final Report on 2010 SBIR Phase I grant DE-SC-0004491

\section{SHORT-PERIOD RF UNDULATOR FOR A SASE NANOMETER SOURCE}

the bigger the diameter, accuracy of a miter bend's angular position should be three times more stringent in comparison with $\mathrm{TE}_{01}-\mathrm{TE}_{02}$ converter positioning. The green line in Fig. 34 shows diffraction losses in ideal miter bend with zero error in inclination.

Corrugation depth in the straight runs within the resonant ring is another important parameter which has to be investigated from the viewpoint of possible tolerances. Changes in corrugation depth lead to changes in propagation constants of the $\mathrm{HE}_{11}$ and $\mathrm{HE}_{12}$ modes. The Fig. 34 is a plot of additional losses due to errors in corrugation depth. In simulations it was assumed that a waveguide section of $250 \mathrm{~mm}$ length has an erroneous corrugation depth. The computational results shown for this case allows conclusion that corrugation depth should be produced with an accuracy of $\pm 0.3 \mathrm{~mm}$.

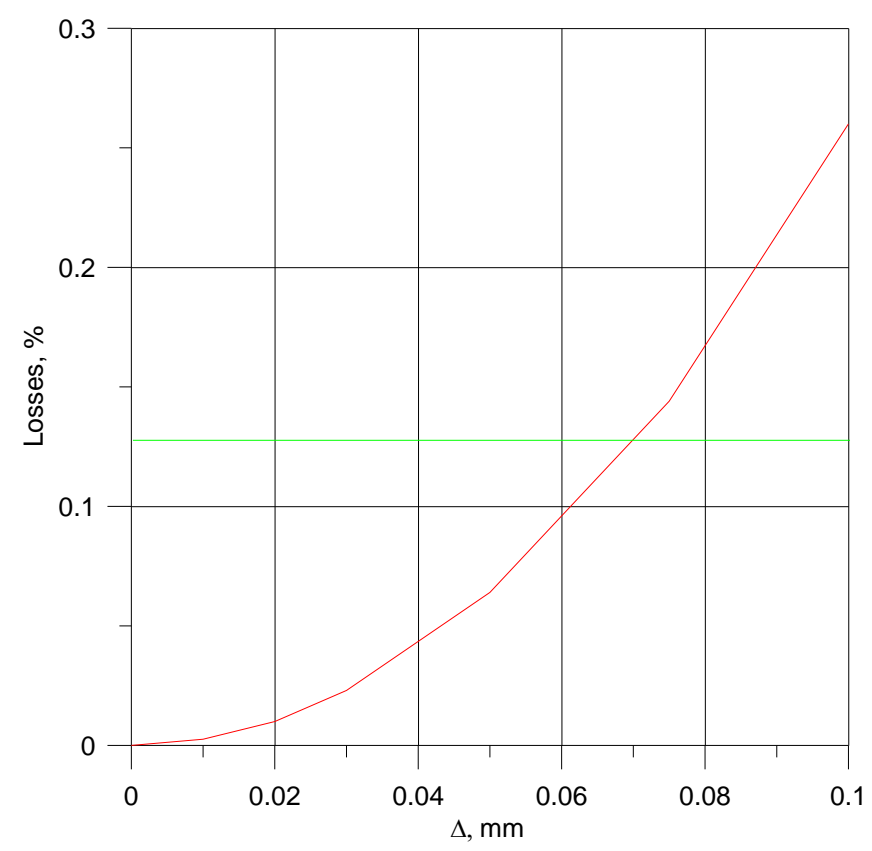

Fig. 34. Additional diffraction losses in resonant ring caused by an error in depth of the corrugation in one of the straight waveguide sections.

Green line shows diffraction losses in a single miter bend.

\section{CONCLUSIONS}

Particle orbit calculations, as described in Section II, show that the presence of a copropagating component of RF field can cause deleterious motion for the undulating electrons that can seriously degrade their radiation spectrum. To obviate this problem, resonator designs were devised in which only the counter-propagating field components interact with the particles. Two resonator configurations with the same undulator parameter $K=0.4$ have parameters summarized in the Table below. Length of the wigglers where an electron beam interacts with a 
Omega-P, Inc.

Final Report on 2010 SBIR Phase I grant DE-SC-0004491

\section{SHORT-PERIOD RF UNDULATOR FOR A SASE NANOMETER SOURCE}

counter-propagating RF wave is $50 \mathrm{~cm}$ in both cases. Other parameters and sizes are taken from Tables II and III.

This Table shows that the $\mathrm{HE}_{11}$ resonant ring has several obvious advantages as compared with the two-mode cavity, however it requires a relatively imposing input power.

\begin{tabular}{|c|c|c|}
\hline & $\begin{array}{c}\mathbf{T E}_{\mathbf{0 1}} / \mathbf{T E}_{\mathbf{0 2}} \text { Mode Conversion } \\
\text { Resonator }\end{array}$ & $\mathbf{H E}_{\mathbf{1 1}}$ Resonant Ring \\
\hline $\begin{array}{c}\text { possibility to change } \\
\text { polarization }\end{array}$ & no & yes \\
\hline input power & $34 \mathrm{MW}(13 \mathrm{MW})$ Note 1 & $120 \mathrm{MW}(85 \mathrm{MW})$ Note 1 \\
\hline pulse duration & $630 \mathrm{~ns}(1660 \mathrm{~ns})$ Note 1 & $700 \mathrm{~ns}(970 \mathrm{~ns})$ Note 1 \\
\hline surface electric field & $0 \mathrm{Mv} / \mathrm{m}$ Note 2 & $20 \mathrm{MV} / \mathrm{m}$ Note 3 \\
\hline surface magnetic field & $0.26 \mathrm{MA} / \mathrm{m}(0.9 \mathrm{MA} / \mathrm{m})$ Note 4 & $0.15 \mathrm{MA} / \mathrm{m}(0.8 \mathrm{MA} / \mathrm{m})$ Note 4 \\
\hline $\begin{array}{c}\text { spurious mode }(\mathrm{co}- \\
\text { propagating to e-beam }) \\
\text { excitation level }\end{array}$ & $-50 \mathrm{~dB}$ & $<-50 \mathrm{~dB}$ Note 5 \\
\hline dominant losses & Ohmic losses & diffraction losses \\
\hline $\begin{array}{c}\text { long RF undulator consisted } \\
\text { of several modules }\end{array}$ & modules in tandem & one long ring is possible \\
\hline
\end{tabular}

Note 1 - Values in case of undulator cooling down to liquid nitrogen temperature.

Note 2 - Surface electric field is not zero at coupling holes: it depends on field enhancement at corner roundings. The transmitted power through each hole is only $2 \mathrm{~kW}$.

Note 3 - Surface field at corrugation when $E$-polarization is parallel to miter bend mirrors (Fig. IIIc-6). In case $E$-polarization is perpendicular to miter bend mirrors, maximum surface field there becomes $\sim 200 \mathrm{MV} / \mathrm{m}$.

Note 4 - Values in brackets are given at mode converters and at miter bend mirrors correspondingly.

Note 5 - In experiments with $\mathrm{TE}_{01}$ resonant rings of similar Q-factor [10] the spurious travelling wave of the opposite rotation was never observed. 
Omega-P, Inc.

Final Report on 2010 SBIR Phase I grant DE-SC-0004491

\section{SHORT-PERIOD RF UNDULATOR FOR A SASE NANOMETER SOURCE}

\section{References}

1. Z. Huang, K.-J. Kim, "Review of x-ray free-electron laser theory," Phys. Rev. ST Accel. Beams 10, 034801 (2007), and copious references therein.

2. C. Pellegrini, "X-Band Microwave Undulators for Short Wavelength Free-Electron Lasers," $27^{\text {th }}$ Int. Free Electron Laser Conference, 21-26 August 2005, Stanford, p. 203.

3. S. Tantawi, V. Dolgashev, C. Nantista, C. Pellegrini, J. Rosenzweig, G. Travish, "A Coherent Compton Backscattering High Gain FEL Using An X-band Microwave Undulator," $27^{\text {th }}$ Int. Free Electron Laser Conference, 21-26 Aug 2005, Stanford, p. 438.

4. R. Talman, “Accelerator X-Ray Sources,” Wiley-VCH, ISBN: 3527405909.

5. T. Shintake, K. Huke, J. Tanaka, I. Sato and I.Kumabe, "Development of microwave undulator", Japanese J. of Appl. Phys., 22, p. 844-851 (1983).

6. M. Seidel, "Parameter Evaluation for Microwave Undulator Schemes", DESY-TESLAFEL Report 2001-08 (2001).

7. S. Tantawi et al, Phys. Rev. ST Accel. Beams 8, 042002 (2005).

8. T.M. Tran, Bruce G. Danly, and J.S. Wurtele, "Free Electron Lasers With Electromagnetic Standing Wave Wigglers", IEEE J. Quantum Electr. QE-23, (1987).

9. R.B. Yoder, T.C. Marshall, and J.L. Hirshfield, "Energy-Gain Measurements from a Microwave Inverse Free-Electron-Laser Accelerator”, Phys. Rev. Lett. 86, 1765 (2001).

10. Bogdashov, A.; Denisov, G.; Lukovnikov, D.; Rodin, Y.; Hirshfield, J., Ka-band resonant ring for testing components for a high-gradient linear accelerator, IEEE Trans. on Microwave Theory and Techniques, 2005, Vol. 53 Issue:10, pp. $3152-3155$. 